\title{
The Einstein Evolution Equations as a First-Order Quasi-Linear Symmetric Hyperbolic System, I
}

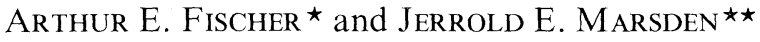 \\ Department of Mathematics, University of California, Berkeley, California, USA \\ Received January 25; in revised form May 4, 1972
}

\begin{abstract}
A systematic presentation of the quasi-linear first order symmetric hyperbolic systems of Friedrichs is presented. A number of sharp regularity and smoothness properties of the solutions are obtained. The present paper is devoted to the case of $\mathbf{R}^{n}$ with suitable asymptotic conditions imposed. As an example, we apply this theory to give new proofs of the existence and uniqueness theorems for the Einstein equations in general relativity, due to Choquet-Bruhat and Lichnerowicz. These new proofs using first order techniques are considerably simplier than the classical proofs based on second order techniques. Our existence results are as sharp as had been previously known, and our uniqueness results improve by one degree of differentiability those previously existing in the literature.
\end{abstract}

\section{§ 0. Introduction}

Part of the folklore of mathematics is that the Friedrich's theory of symmetric hyperbolic systems extends to the quasi-linear case. Our original motivation for looking at these systems came from the fact that it is possible to reduce the Einstein system studied by Choquet-Bruhat and Lichnerowicz $[3,4,33]$ to a first order symmetric hyperbolic system. The techniques these authors used are based on the second order theory of Leray [32] as improved by Dionne [16].

However we needed a version of the symmetric hyperbolic theory with sharper differentiability properties than previously existed in the literature. The basic theory is presented in $\$ \S 1,2$ below. We consider the equations in $\boldsymbol{R}^{n}$ with asymptotic conditions imposed. Presumably similar results are true for bounded regions with suitable boundary conditions. One could also argue locally in $\boldsymbol{R}^{n}$ and use domain of dependence arguments; cf. Fischer-Marsden [21], Wilcox [42]. Part II will deal with the theory on manifolds.

This theory is complicated in its technical details by two facts. First, differentiability properties of the coefficients complicate the proof that the solutions are just as differentiable as the initial data in the Sobolev class $H^{s}$. Second, we want this value of $s$ to be the best possible, $s>n / 2+1$.

$\star$ Partially supported by AEC Contract AT(04-3)-34.

$\star \star$ Partially Supported by NSF Contract GP-8257.

1 Commun. math. Phys., Vol. 28 
The linear case is presented in $\S 1$. For the applications, we want to make the assumptions on the coefficients as minimal as possible. This necessitates using carefully the domain of the linear generator of the equations. In particular the usual energy estimates are not good enough to show that the top order derivatives are well behaved. This is replaced by a perturbation argument. This is then used in $\S 2$ to effect the quasilinear case. The proof is, in outline, that suggested by the standard method of handling quasi-linear equations (cf. Courant-Hilbert [15]). Namely, one sets up a suitable contraction mapping on a function space using the linear theory, and the fixed point represents the solution. It is propagated only for a small $t$-interval.

Several properties are established in addition to the existence and uniqueness theory. For example we establish regularity (the time of existence does not depend on $s$, the differentiability degree) -- this was also obtained for the second order systems by Choquet-Bruhat $[7,9]$. We also show that the propagator $U_{t}(u), u \in H^{s}$, is a $C^{\infty}$ function of $u$ for fixed $t$ and is jointly continuous in $t, u$.

We go on in $\S \S 3,4$ to apply these results to the Einstein system. As in $[3,33]$ we use harmonic coordinates. The key idea is that in these coordinates the top order terms in the Einstein system uncouple. In the second order theory this enables one to verify strict hyperbolicity; here it enables one to reduce the equations to a symmetric hyperbolic system in the same way that one reduces the wave equation to a symmetric hyperbolic system.

We obtain most of the important results in a fairly compact exposition, using the relatively simple theory of symmetric hyperbolic systems. We deal directly with the asymptotic conditions, obtain the best possible value $s \geqq 4$, and establish uniqueness of Ricci flat $H^{s}$ spacetimes up to $H^{s+1}$ coordinate transformations (in [6] it is $H^{s}$ ). Results local in space can be dealt with in the same manner; cf. [21].

\section{§1. Linear Symmetric Hyperbolic Systems}

A symmetric hyperbolic system in Euclidean space has the form

$$
A^{0}(t, x) \frac{\partial u}{\partial t}=\sum_{i=1}^{n} A^{i}(t, x) \frac{\partial u}{\partial x^{i}}+B(t, x) u+C(t, x)
$$

where $A^{0}(t, x)$ is an $m \times m$ positive definite symmetric matrix for $(t, x) \in \boldsymbol{R} \times \boldsymbol{R}^{n}, u=u(t, x)=u_{t}(x)$ is an $m$ component vector and $A^{i}(t, x)$ are symmetric matrices.

These systems have been studied and applied rather extensively. We refer, for example to Friedrichs [23], Lax [31], Courant-Hilbert [15], 
Dunford-Schwartz [17], Phillips [37], and Kato [29]. See also Wilcox [42] and Schulenberger and Wilcox [38].

In this section we would like to present a few refinements of theorems due to the aforementioned authors. These refinements concern the differentiability of the coefficients and are needed in the quasi-linear theory $(\S 2)$ and in subsequent applications.

We denote by $H^{s}\left(\boldsymbol{R}^{n}, \boldsymbol{R}^{m}\right)=H^{s}$, the usual Sobolev space; $H^{s}$ is the completion of the $C^{\infty}$ functions with compact support under the $H^{s}$ norm:

$$
\|f\|_{s}^{2} \equiv\|f\|_{H^{s}}^{2} \equiv \sum_{0 \leqq|k| \leqq s} \int\left|D^{k} f(x)\right|^{2} d x .
$$

Here $D^{k} f$ denotes the total derivative of order $k$.

We make use of two basic Sobolev-type theorems:

(i) $H^{s}$ is a ring under multiplication,

$$
\|f \cdot g\|_{s} \leqq \text { const }\|f\|_{s}\|g\|_{s}
$$

if $s>n / 2$. Here $f \cdot g$ stands for some bilinear form from $\boldsymbol{R}^{m} \times \boldsymbol{R}^{m^{\prime}} \rightarrow \boldsymbol{R}^{m^{\prime \prime}}$ composed with $f \times g$; for example, componentwise multiplication;

(ii) for $s>\frac{n}{2}+k, H^{s} \subset C^{k}$, and

$$
\|f\|_{C^{k}} \leqq \text { const }\|f\|_{s}
$$

where $\|f\|_{C^{k}}$ is the supremum of $|f|,|D f|, \ldots,\left|D^{k} f\right|$ over all of $\boldsymbol{R}^{n}$.

These results are standard; see for instance Nirenberg [35].

Our starting point will be a result which is essentially contained in the above references. The assumptions are as follows:

(i) $s>n / 2+1$.

(ii) $A^{0}$ is symmetric positive definite and

$$
t \mapsto A_{t}^{0}(x)-I \equiv A^{0}(t, x)-I
$$

is a $C^{1}$ map from $\boldsymbol{R}$ to $H^{s}\left(\boldsymbol{R}^{n}, \boldsymbol{R}^{m^{2}}\right)$ and a $C^{0}$ map from $\boldsymbol{R}$ to $H^{s+1}\left(\boldsymbol{R}^{n}, \boldsymbol{R}^{m^{2}}\right)$. Here $I$ is the $m \times m$ identity matrix.

(iii) $A^{i}$ is symmetric, $i=1, \ldots, n$ and

$$
t \mapsto A_{t}^{i}(x)=A^{i}(t, x)
$$

is a $C^{0}$ map from $\boldsymbol{R}$ to $H^{s}\left(\boldsymbol{R}^{n}, \boldsymbol{R}^{m^{2}}\right)$.

(iv) $t \mapsto B_{t}(x)=B(t, x)$ is a continuous curve from $\boldsymbol{R}$ to $H^{s}\left(\boldsymbol{R}^{n}, \boldsymbol{R}^{m^{2}}\right)$.

(v) $t \mapsto C_{t}(x)=C(t, x)$ is a continuous curve from $\boldsymbol{R}$ to $H^{s}\left(\boldsymbol{R}^{n}, \boldsymbol{R}^{m}\right)$.

1.1. Theorem. Let assumptions $A_{1}$ hold. For any $u_{0} \in H^{s}$ there is a unique continuous curve $t \mapsto u_{t} \in H^{s}$ which is differentiable as a curve in $1^{*}$ 
$H^{s-1}$ (in the norm topology) and equals $u_{0}$ at $t=0$ and which satisfies Eq. (1). (Note that each side of Eq. (1) is an element of $\mathrm{H}^{\mathrm{s}-1}$.)

In fact there is a family of smooth maps

such that

$$
U(t, s): H^{s} \rightarrow H^{s}, \quad t, s \in \boldsymbol{R}
$$

(i) $U(t, s) \circ U(s, r)=U(t, r)$ (Chapman-Kolmogorov equation),

(ii) $U(t, t) u_{0}=u_{0}$, and

(iii) $t \mapsto U(t, s) u_{0}$ is a continuous curve in $H^{s}$ and a $C^{1}$ curve in $H^{s-1}$ and satisfies Eq. (1). We refer to $U(t, s)$ as the flow defined by Eq. (1).

This result is generally attributed to Friedrichs.

Remark. The solution $u(t, x)$ will, from the differential equation itself, be locally jointly $H^{s}$ in $(t, x)$. However, that $t \mapsto u_{t}$ is a $C^{0}$ curve in $H^{s}$ and a $C^{1}$ curve in $H^{s-1}$ is saying more. Indeed, from the restriction theorem (Palais [36]), if $u(t, x)$ is jointly $H^{s}$, it does not have to be $H^{s}$ in $x$ for fixed $t$ (one "loses $\frac{1}{2}$ a derivative" on restriction to a hyperplane).

Since Theorem 1.1 does not appear in the above form in the literature, we shall sketch its proof.

\section{Sketch of Proof}

Since $A^{0}$ is symmetric and positive definite, we can write $A^{0}=T^{2}$ where $T$ is symmetric and positive definite. Moreover $T-I$ will be in $H^{s+1}$ and $C^{1}$ as a curve in $H^{s}$. Thus $T$ is close to $I$ at spatial infinity, and so $T$ or $T^{-1}$ multiplied with something in $H^{s}$ is again $H^{s}$ (write $T A=(T-I) A+A)$. Then $v=T u$ satisfies (using the summation convention on repeated indices)

$\frac{\partial v}{\partial t}=T^{-1} A^{i} T^{-1} \frac{\partial v}{\partial x^{l}}+\left[T^{-1} A^{i} \frac{\partial T^{-1}}{\partial x^{i}}+T \frac{\partial T}{\partial t} T^{-1}+T^{-1} B T^{-1}\right] v+T^{-1} C$

which is of the form

$$
\frac{\partial v}{\partial t}=\tilde{A}^{i} \frac{\partial v}{\partial x^{i}}+\tilde{B} v+\tilde{C}
$$

where now $\tilde{A}^{i}, \tilde{B}, \tilde{C}$ satisfy the same hypotheses with $\tilde{A}^{0}=I$. Thus this change of variables reduces the problem to $A^{0}=I$. Note that one degree of differentiability was lost in the transformation. If $A^{0}$ is only $H^{s}$, different arguments are needed - see below. This change of variables may be found in Courant-Hilbert [15].

We see from our assumptions together with Sobolev's theorem that the conditions in Kato [29] are met. This assures the existence of unique $t$-continuous solutions in $H^{1}$, and $t$-differentiable in $L_{2}$. 
To obtain the $H^{s}$ case from this we employ the Leray energy estimates, as in Courant-Hilbert [15]. First we proceed formally; letting (, ) be the $L_{2}$ inner product, we have

So

$$
\begin{aligned}
\frac{1}{2} \frac{\partial}{\partial t}(v, v) & =\left(\tilde{A}^{i} \frac{\partial v}{\partial x^{i}}, v\right)+(\tilde{B} v, v)+(\tilde{C}, v) \\
& =-\frac{1}{2}\left(\left(\frac{\partial \tilde{A}^{i}}{\partial x^{i}}\right) v, v\right)+(\tilde{B} v, v)+(\tilde{C}, v) \\
& \leqq \text { constant }(v, v)+\text { constant }\|v\|_{L_{2}}, \quad-T \leqq t \leqq T .
\end{aligned}
$$

$$
\left\|v_{t}\right\|_{L_{2}} \leqq e^{k_{T} t}\left\|v_{0}\right\|_{L_{2}}, \quad-T \leqq t \leqq T .
$$

Here we have written

$$
\left(\tilde{A}^{i} \frac{\partial v}{\partial x^{i}}, v\right)=\int \frac{\partial}{\partial x^{i}}\left(\tilde{A}^{i} v \cdot v\right) d x-\int\left(\frac{\partial}{\partial x^{i}} \tilde{A}^{i}\right) v \cdot v d x-\int \tilde{A}^{i} v \cdot \frac{\partial v}{\partial x^{i}} d x
$$

and exploited symmetry of $\tilde{A}^{i}$. We thus have $\|v\|_{L_{2}}$ bounded on finite $t$-intervals. Note $\frac{\partial \tilde{A}^{i}}{\partial x^{i}}$ is uniformly bounded as $s>n / 2+1$.

We continue this process up through the $H^{s}$ norm. One must exploit the ring property of $H^{s}$ here, being slightly careful as our coefficients are just $H^{s}$. Consider the total derivative of order $s$; again we have, formally,

$$
\begin{aligned}
\frac{1}{2} \frac{\partial}{\partial t}\left(D^{s} v, D^{s} v\right)= & \left(D^{s}\left(\tilde{A}^{i} \frac{\partial v}{\partial x^{i}}+B v+C\right), D^{s} v\right) \\
= & \left(\tilde{A}^{i} \frac{\partial}{\partial x^{i}}\left(D^{s} v\right)+\cdots+\left(D^{s} \tilde{A}^{i}\right) \frac{\partial v}{\partial x^{i}}, D^{s} v\right) \\
& +\left(\left(D^{s} B\right) v+\cdots+B D^{s} v, D^{s} v\right)+\left(D^{s} C, D^{s} v\right) .
\end{aligned}
$$

The first term $\left(\tilde{A}^{i} \frac{\partial}{\partial x^{i}}\left(D^{s} v\right), D^{s} v\right)$ may be dealt with as in the $L_{2}$ case above. The other terms are handled by Sobolev inequalities. For example

$$
\begin{aligned}
\left(D^{s} \tilde{A}^{i} \frac{\partial v}{\partial x^{i}}, D^{s} v\right) & \leqq\left\|\frac{\partial v}{\partial x^{i}}\right\|_{C^{0}}\left\|D^{s} \tilde{A}\right\|_{L_{2}}\left\|D^{s} v\right\|_{L_{2}} \\
& \leqq(\text { const })\|v\|_{H^{s}}^{2}\|\tilde{A}\|_{H^{s}}
\end{aligned}
$$

The rest of the intermediate terms are handled in a similar way as in the proof that $H^{s}$ is a ring. The $B$ terms come immediately from the ring property:

$$
\begin{aligned}
\left(D^{s}(B v), D^{s} v\right) & \leqq\|B v\|_{H^{s}}\|v\|_{H^{s}} \\
& \leqq \text { const }\|B\|_{H^{s}}\|v\|_{H^{s}}^{2} .
\end{aligned}
$$

Warning: This technique does not work if $B$ is only $H^{s-1}$. 
These arguments show that there is an a priori bound on the $H^{s}$ norm. To make the proof precise requires further arguments. Indeed, at first one doesn't even know that $D^{s} v$ is an $L_{2}$-function. This can be remedied by a $C^{\infty}$ approximation argument. Namely, we observe that we would rigorously obtain the above bounds if $v$ were $C^{\infty}$. So approximate the coefficients by $C^{\infty}$ ones and the initial data by $C^{\infty}$ data. By differentiating the equation it is not hard to see that the solution is $C^{\infty}$ (or use DunfordSchwartz [17]), and by the $H^{s}$ estimates above, our sequence of approximating $C^{\infty}$ solutions converges in $H^{s}$. The theorem then follows.

Theorem 1.1 is not yet sharp enough for the applications we have in mind. We wish to allow $B$ to be just $H^{s^{-1}}$ and $A^{0}$ to be $H^{s}$. Let us observe that without special conditions on $A^{i}, H^{s-1}$ solutions need not be $H^{s}$ if the initial data is $H^{s}$. Indeed, take the case $A^{0}=I, A^{i}=0, C=0$ and $m=1$. Then we are dealing with the equation $\frac{\partial u}{\partial t}=B u$. This has solution $u(t, x)=e^{\int_{0}^{t} B d t} u_{0}(x)$ which is only $H^{s-1}$ if $B$ is only $H^{s-1}$.

To deal with this case, we consider first a few lemmas:

1.2. Lemma. Let $A$ be the generator of a $C^{0}$ semi-group $e^{t A}$ on a Banach space $\mathscr{E}$ with $D_{A}$ the domain of $A$. Let $D \subset D_{A}$ be a Banach space continuously included in $D_{A}$ (with the graphtnorm). Suppose $e^{t A}$ leaves $D$ invariant and forms a $C^{0}$ semi-group there. If $D$ is dense in $D_{A}$, then $D$ is a core for $A$ (cf. Kato [26] for terminology).

Proof. The argument is along standard lines; cf. Kato [29], p. 243. Namely, from

$$
(\lambda-A)^{-1}=\int_{0}^{\infty} e^{-\lambda t} e^{t A} d t
$$

for sufficiently large $\lambda$, we see that $(\lambda-A)^{-1}$ maps $D$ to $D$. Now $(\lambda-A)^{-1}$ is one to one and maps $\mathscr{E}$ onto $D_{A}$. Since $D$ is dense in $\mathscr{E}$, the image $(\hat{\lambda}-A)^{-1}(D)$ is also dense as is easily seen using $\mathscr{E} *$. From this it follows easily that $A$ is the closure of $A \uparrow D$ as required.

Further details and applications of results like this are found in Chernoff-Marsden [14].

The purpose of this lemma is to insure that when we regard $A^{i} \frac{\partial u}{\partial x^{i}}$ as an operator from $H^{s}$ to $H^{s-1}$, its closure is the generator of a (semi-) group and hence the correct domain may be obtained from this closure. We state this formally as follows:

1.3. Corollary. Let $A_{t} u=\Sigma A^{i} \frac{\partial u}{\partial x^{i}}$ where $A^{i}$ satisfies conditions $A_{1}$ (iii). Regard $A_{t}$ as an operator from $H^{s}$ to $H^{s-1}$. Then the closure of $A_{t}$, for 
each $t$, is a generator of a one parameter group. Let this closure be $\bar{A}_{t}$ with domain $D_{t}$.

Proof. Fix $t$. Then by Theorem 1.1, $A_{t}$ generates a flow on $L_{2}$ which maps $H^{s}$ to $H^{s}$, and $H^{s-1}$ to $H^{s-1}$. The generator of this flow, restricted to $H^{s-1}$ is clearly an extension of $A_{t}: H^{s} \rightarrow H^{s-1}$. Now apply the lemma with $\mathscr{E}=H^{s-1}$ and $D=H^{s}$.

1.4. Lemma. Let $\bar{A}_{t}: D_{t} \subset \mathscr{E} \rightarrow \mathscr{E}$ be the generator of a flow $U(t, s): \mathscr{E} \rightarrow \mathscr{E}$. Let $t \mapsto B_{t}$ be a strongly continuous curve of bounded operators on $\mathscr{E}$. Then $\bar{A}_{t}+B_{t}$ has a (unique) flow, $V(t, s)$.

Proof. This is proved as in Kato [27], Theorem 4.5.

Warning. The flow $V(t, s)$ need not map the domain $D_{s}$ to the domain $D_{t}$. Since $B_{t}$ is a bounded operator, the domain of $\bar{A}_{t}$ is the same as that of $\overline{A_{t}}+B_{t}$.

If we apply these remarks to symmetric hyperbolic systems we obtain a result in which $B$ need only be $H^{s-1}$. Our assumptions are as follows:

(i) $s>n / 2+1$.

(ii) $A^{0}$ is symmetric positive definite and $t \mapsto A_{t}^{0}(x)-I$ is a $C^{1}$ map from $\boldsymbol{R}$ to $H^{s-1}$ and a $C^{0}$ map from $\boldsymbol{R}$ to $H^{s}$.

(iii) $A^{i}$ is symmetric and $t \mapsto A^{i}$ is a $C^{0}$ map from $\boldsymbol{R}$ to $H^{s}$.

(iv) $t \mapsto B_{t}(x)$ is a continuous curve from $\boldsymbol{R}$ to $H^{s-1}$.

(v) $t \mapsto C_{t}(x)$ is a continuous curve from $\boldsymbol{R}$ to $H^{s-1}$.

1.5. Theorem. Let assumptions $A_{2}$ hold. Let $D_{s}$ denote the domain of the closure of the operator

$$
A_{t}(u)=\Sigma A^{i}(t, x) \frac{\partial u}{\partial x^{i}}
$$

as a map of $H^{\mathrm{s}}$ to $\mathrm{H}^{\mathrm{s}-1}$. Assume $D_{\mathrm{s}}$ is independent of $t$.

Then for $u_{0} \in D_{s}$ there is a unique solution $u_{t} \in D_{s}$ to

$$
A^{0}(t, x) \frac{\partial u}{\partial t}=\sum_{i=1}^{n} A^{i}(t, x) \frac{\partial u}{\partial x^{i}}+B_{t} u+C_{t} .
$$

In fact this equation defines a flow $U(t, s): H^{s-1} \rightarrow H^{s-1}$ which maps $D_{\mathrm{s}}$ to $D_{\mathrm{s}}$.

Proof. We may assume, as in Theorem 1.1, that $A^{0}$ is the identity. Applying 1.4 we see that

has a well defined flow.

$$
\frac{\partial v}{\partial t}=\Sigma \tilde{A}^{i} \frac{\partial v}{\partial x^{i}}+\tilde{B} v+\tilde{C}
$$

If the inhomogeneous term were $H^{s}$, there would be no problem, as in Kato [29], Theorem 7.1. However, even for $\tilde{C}$ only $H^{s-1}$ we claim 
that a solution in the domain of $A_{t}$ remains in the domain. This is not obvious at all from Duhamel's formula (which masks extra "smoothing"). However, the result does follow from a more general smoothness result on lipschitzian perturbations of linear systems due to Segal [39]. It applies in particular here to show that initial data in the domain of $\bar{A}$ remains in the domain. See also Chernoff-Marsden [14] for an exposition and further applications of Segal's result.

That we may allow $\tilde{C}$ to be just $H^{s-1}$ will be important below in studying asymptotic conditions.

Warning. For the hypotheses $\left(\mathrm{A}_{2}\right)$, the energy estimates only hold up through the $H^{s-1}$ norm. The added differentiability comes from the domain of $A_{t}$. It is not in general true that $H^{s}$ initial data remains $H^{s}$.

Next we proceed to consider linear symmetric hyperbolic systems with asymptotic conditions imposed.

That $u \in H^{s}\left(\boldsymbol{R}^{n}, \boldsymbol{R}^{m}\right)$ means that $u$ dies off to zero fairly quickly at $\infty$. In particular for $s>n / 2,|u(x)| \rightarrow 0$ uniformly as $|x| \rightarrow \infty$. For relativity we wish to impose a different condition at $\infty$; for example $u$ is asymptotically constant. More precisely, we make:

Definition. Fix $w: \boldsymbol{R}^{n} \rightarrow \boldsymbol{R}^{m}$. We say a map $u: \boldsymbol{R}^{n} \rightarrow \boldsymbol{R}^{m}$ is $H^{\text {s }}$ asymptotic to $w$ iff $u-w \in H^{s}\left(\boldsymbol{R}^{n}, \boldsymbol{R}^{m}\right)$. We denote this set of $u^{\text {'s }}$ by $H_{w}^{s}\left(\boldsymbol{R}^{n}, \boldsymbol{R}^{m}\right)$. By translation, $H_{w}^{s}$ inherits a metric structure.

1.6. Lemma. Let $s>n / 2$ and suppose the derivatives of $w$ of order $\leqq s$ are continuous and bounded on all of $\boldsymbol{R}^{n}$. Then (componentwise) multiplication

is a continuous map.

$$
H_{w}^{s} \times H^{s} \rightarrow H^{s}
$$

Proof. A typical element of $H_{w}^{s}$ is $f+w$ where $f \in H^{s}$. Now for $g \in H^{s}$, writing

$$
(f+w) g=f g+w g
$$

we notice that $f g \in H^{s}$ as $H^{s}$ is a ring, and $w g \in H^{s}$ since the derivatives of $w$ are uniformly bounded. Thus for $f_{1}, g_{1} \in H^{s}$

$$
\left\|\left(f_{1}+w\right) g_{1}-(f+w) g\right\|_{s} \leqq\left\|f g-f_{1} g_{1}\right\|_{s}+\text { const }\left\|g-g_{1}\right\|_{s}
$$

so the lemma follows.

In particular, multiplication by $f \in H_{w}^{s}$ is a continuous linear map of $H^{s}$ to $H^{s}$.

We now consider again our system (1):

$$
A^{0}(t, x) \frac{\partial u}{\partial t}=\sum_{i=1}^{n} A^{i}(t, x) \frac{\partial u}{\partial x^{i}}+B(t, x) u+C(t, x) .
$$


Let $C_{b}^{s}$ denote the Banach space of maps which are of class $C^{s}$ and have bounded derivatives of order $\leqq s$. We formulate our assumptions as follows:

(i) $s>n / 2+1$.

(ii) $A_{t}^{0} \in H_{\widetilde{A}_{t}^{0}}^{s}$ where $t \mapsto \tilde{A}_{t}^{0} \in C_{b}^{s}$ is a $C^{0}$ curve and a $C^{1}$ curve to $C_{b}^{s-1}$. Moreover, $A_{t}^{0}$ is symmetric and positive definite with, more specifically,

$$
\left\langle A_{t}^{0} x, x\right\rangle \geqq c_{t}\langle x, x\rangle
$$

where $c_{t}>0$ is a constant depending continuously on $t$. (The latter assumption then also follows for $\tilde{A}_{t}^{0}$.)

Also assume $t \mapsto A_{t}^{0}-\tilde{A}_{t}^{0} \in H^{s}$ is a $C^{0}$ curve and a $C^{1}$ curve into $H^{s-1}$.

(iii) $A_{t}^{i} \in H_{\tilde{A}_{t}^{\prime}}^{s}$ where $t \mapsto \tilde{A}_{t}^{i} \in C_{b}^{s}$ is a $C^{0}$ curve and $t \mapsto A_{t}^{i}-\tilde{A}_{t}^{i} \in H^{s}$ is a $C^{0}$ curve. Also, $A_{t}^{i}$ are symmetric and $D_{s}$ is independent of $t$.

(iv) $B_{t} \in H_{\tilde{B}_{t}}^{s-1}$ where $t \mapsto \tilde{B}_{t} \in C_{b}^{s-1}$ is a $C^{0}$ curve and $t \mapsto B_{t}-\tilde{B}_{t} \in H^{s-1}$ is a $C^{0}$ curve.

(v) $t \mapsto C_{t}$ is a $C^{0}$ curve in $H^{s-1}$.

(vi) Let $w \in C_{b}^{s}$ and assume (a) $\frac{\partial w}{\partial x^{j}} \in H^{s-1}$ and (b) $B_{t} \cdot w \in H^{s-1}$.

Note. The condition (vi) is not the same as $w \in H^{s}$ and the distinction will be important later for the Schwarschild type of asymptotic condition we want to impose.

1.7. Theorem. Let assumptions $\left(\mathrm{A}_{3}\right)$ hold and let $D_{s}$ denote the domain of $\bar{A}_{t}=\overline{\left(A_{t}^{i} \frac{\partial}{\partial x^{i}}\right)}$ as an operator from $H^{s}$ to $H^{s-1}$. Then system (1) has a unique solution $u(t) \in D_{s}+w \subset H_{w}^{s-1}$ for $u(0) \in D_{s}+w$. Indeed, Eq. (1) defines a flow on $H_{w}^{s-1}$ with domain $D_{s}+w$ for its generator. If $s>n / 2+k$, $u(t) \in C_{b}^{k-1}$.

Proof. Observe that the equation for $v=u-w$ is

$$
A^{0} \frac{\partial v}{\partial t}=A^{i} \frac{\partial v}{\partial x^{i}}+B v+\left(A^{i} \frac{\partial w}{\partial x^{i}}+B w+C\right) .
$$

By Lemma 1.6 and assumptions (v), (vi), the inhomogeneous term is $H^{s-1}$. Now we assert, as before, that we can assume $A^{0}=$ Identity. This is done just as before. The assumption concerning $A_{t}^{0}$ is necessary to insure that $T^{-1}$ remains bounded on all of $\boldsymbol{R}^{n}$ so that the transformed equation will have $B$ satisfying the same assumptions. So we can assume $A^{0}=$ Identity. 
Now we first consider

$$
\frac{\partial v}{\partial t}=A_{t}^{i} \frac{\partial v}{\partial x^{i}} .
$$

Since $A_{t}^{i} \in H_{\tilde{A}_{t}^{t}}^{s}$ and $s>n / 2+1$, and $\tilde{A}_{t}^{i} \in C_{b}^{s}$, it follows that the $C^{1}$ norm of $A_{t}^{i}$ is bounded. Thus $t \mapsto A_{t}^{i} \in C_{b}^{1}$ is continuous. Thus by Kato [29], as before, this equation has unique solutions in $H^{1}$ and $L_{2}$. The point now is that because of Lemma 1.6, multiplication by $A_{t}^{i}$ is still continuous from $H^{s}$ to $H^{s}$ so the Leray energy argument will still work. Thus we get a solution flow to $\partial v / \partial t=A_{t}^{i} \partial v / \partial x^{i}$ in $H^{s}$.

We can, as before, add on the $B$ term because by Lemma 1.6, $B: u \mapsto B u$ is a bounded operator on $H^{s-1}$. Thus we get solutions to

$$
\frac{\partial v}{\partial t}=A_{t}^{i} \frac{\partial v}{\partial x^{l}}+B v
$$

in the domain of the closure of the operator $A_{t}^{i} \frac{\partial}{\partial x^{i}}$.

Since the inhomogeneous term is $H^{s-1}$, we can, as before, add it on as well. Thus we get our solution $u=v+w$.

Finally, in this section, we shall present an example of how this theory can be used to prove a sharp result for the wave equation. The result actually goes back to Sobolev (see [40], § 21 where different techniques are used). We shall generalize it to include asymptotic conditions as well. This result will be used in $\S 4$ to obtain the best possible differentiability results on spacetime coordinate transformations connecting two solutions of the Einstein equations.

Basically we want to prove here that solutions to the wave equation with $H^{s}, H^{s-1}$ coefficient functions preserve $H^{s+1}, H^{s}$ Cauchy data. Let $\mathbf{1}=\eta_{\mu \nu}$ denote the Minkowski matrix on $\boldsymbol{R}^{4}$, let $m \geqq 0$ be given, and let $\mathbf{1}_{m}$ be equal to $\mathbf{1}$ plus a term of class $C_{b}^{s}$ which is $\frac{2 m}{r} \boldsymbol{I}$ outside of a bounded region in $\boldsymbol{R}^{3}$, where $\boldsymbol{I}=\delta_{\mu \nu}$ is the unit $4 \times 4$ matrix. Thus for $r$ sufficiently large,

$$
\mathbf{1}_{m}=\eta_{\mu \nu}+\frac{2 m}{r} \delta_{\mu \nu}=\left(\begin{array}{cccc}
-1+\frac{2 m}{r} & 0 & 0 & 0 \\
0 & 1+\frac{2 m}{r} & 0 & 0 \\
0 & 0 & 1+\frac{2 m}{r} & 0 \\
0 & 0 & 0 & 1+\frac{2 m}{r}
\end{array}\right) \text {, }
$$


which is just the asymptotic Schwarschild metric in isotropic coordinates on a $t=$ constant hypersurface. Let $\mathscr{L}^{s} \subset H_{\mathbf{1}_{m}}^{s}$ denote the maps from $\boldsymbol{R}^{3}$ to the Lorentz matrices on $\boldsymbol{R}^{4}$ which are $H^{s}$ asymptotic to $\mathbf{1}_{m}$; we call such maps asymptotically flat with mass $m$ (see p. 23).

1.8. Theorem. Let $s>n / 2+1, n=3$. Let $g_{\mu \nu}\left(t, x^{l}\right)$ be a given Lorentz metric on $\boldsymbol{R}^{4}$ (or $(-\varepsilon, \varepsilon) \times \boldsymbol{R}^{3}$ ) satisfying the conditions:

(i) $t \mapsto g_{\mu v}(t, \cdot)$ is a $C^{0}$ curve in $\mathscr{L}^{s}$ and a $C^{1}$ curve in $H_{\mathbf{1}_{m}}^{s-1}$.

(ii) The $t=$ constant surfaces are space-like for $g_{\mu v}$; specifically, assume $g_{i j} \xi^{i} \xi^{i} \geqq c\|\xi\|^{2} \xi \in \boldsymbol{R}^{3}, c>0$ fixed, and $-g_{00}(t, x) \geqq \delta>0$.

(iii) Let $b^{\mu}(t, x)$ be a $C^{0}$ curve in $H_{\tilde{b}}^{s-1}$ where $\tilde{b}^{\mu} \in C_{b}^{s}$ and $c(t, x), d(t, x)$ are $C^{0}$ curves in $H^{s-1}$ and $H_{\widetilde{d}}^{s-1}$ respectively, where $\tilde{d} \in C_{b}^{s}$.

(iv) Let $w \in C_{b}^{s}\left(\boldsymbol{R}^{3}, \boldsymbol{R}\right)$ with $\frac{\partial w}{\partial x^{i}} \in H^{s-1}$ and let $\left(\psi_{0}, \dot{\psi}_{0}\right) \in H_{w}^{s+1}\left(\boldsymbol{R}^{3}, \boldsymbol{R}\right)$ $\times H^{s}\left(\boldsymbol{R}^{3}, \boldsymbol{R}\right)$ (note that $H^{s}\left(\boldsymbol{R}^{3}, \boldsymbol{R}\right)$ is the tangent space to $H_{w}^{s}\left(\boldsymbol{R}^{3}, \boldsymbol{R}\right)$ ).

Then there exists a unique $\psi(t, x)$, a $C^{0}$ curve in $H_{w}^{s+1}\left(\boldsymbol{R}^{3}, \boldsymbol{R}\right)$ and a $C^{1}$ curve in $H_{w}^{s}\left(\boldsymbol{R}^{3}, \boldsymbol{R}\right)$ with derivative in $H^{s}\left(\boldsymbol{R}^{3}, \boldsymbol{R}\right)$ and which is also locally jointly $H^{s+1}$ in $t, x$ such that

and

$$
\left(\psi(0, x), \frac{\partial \psi}{\partial t}(0, x)\right)=\left(\psi_{0}(x), \dot{\psi}_{0}(x)\right)
$$

$$
g^{\mu \nu}(t, x) \frac{\partial^{2} \psi}{\partial x^{\mu} \partial x^{v}}+b^{\mu}(t, x) \frac{\partial \psi}{\partial x^{\mu}}+c(t, x) \psi+d(t, x)=0 .
$$

Proof. We can apply Theorem 1.7. One can easily define $A^{0}, A^{i}, B, C$ (see $\S 3$ below and Courant-Hilbert [15], p. 595), so that our equation becomes the symmetric hyperbolic system $A^{0} \partial u / \partial t=\Sigma A^{j} \partial u / \partial x^{j}+B u+C$ in the five component vector

$$
u=\left(\begin{array}{l}
\psi \\
\psi_{, l} \\
\psi_{, 0}
\end{array}\right)
$$

We have enough differentiability so that $\psi_{, i}=\partial \psi / \partial x^{i}$ is the classical derivative. (See note on p. 38.)

We shall now check out the assumptions $\left(\mathrm{A}_{3}\right)$ of Theorem 1.7. Conditions (i) -(v) all hold by assumption, with the asymptotic values $\tilde{A}^{0}, \tilde{A}^{i}$ given by inserting the metric $\mathbf{1}_{m}$ into $A^{0}, A^{i}$. The $w$ of (vi) is given by $\left(\begin{array}{l}w \\ 0 \\ 0\end{array}\right)$. The special assumption $B \cdot w \in H^{s-1}$ holds by virtue of the fact that $c \in H^{s-1}$, i.e., $c$ is asymptotic to zero.

Next we shall determine the domain of the operator $A^{j} \frac{\partial u}{\partial x^{j}}=A \cdot u$. 
1.9. Lemma. The domain of the closure of $A$ as an operator from $H^{s}$ to $H^{s-1}$ is given on the three blocks of $u$ by

where

$$
D=H^{s-1} \oplus G^{s} \oplus H^{s}
$$

$$
G^{s}=\left\{\phi_{, i} \mid g^{i j} \frac{\partial \phi_{, i}}{\partial x^{j}} \in H^{s-1}\right\} \text {. }
$$

Proof. It suffices to treat the three blocks separately. On the first block $A$ is the zero operator (the first column of each $A^{j}$ is zero) so that it clearly extends to all of $H^{s-1}$.

On the second block, $A$ is the operator

$$
\phi, j \mapsto\left(0,0,0,0, g^{i j} \frac{\partial \phi, j}{\partial x^{i}}\right) .
$$

Thus $A$ extends to $G^{s}$. Then it is easy to see that on this domain $A$ is closed (that is, $u_{n} \in G^{s}, u_{n} \rightarrow u$ in $H^{s-1}, A u_{n} \rightarrow v$ in $H^{s-1}$ implies $u \in G^{s}$ and $A u=v$ ). Now the closure of $A$ on $H^{s}$ has domain $G^{s}$ since $A$ on $G^{s}$ is a closed extension, and we know from Lemma 1.2 that $H^{s}$ is a core for $A$ in $H^{s-1}$ (the corresponding flow leaves $H^{s}$ invariant).

Finally, on the last block of $u, A$ is the operator

$$
\phi \mapsto\left(0, g^{i j} \frac{\partial \phi}{\partial x^{j}}, 2 g^{0 j} \frac{\partial \phi}{\partial x^{j}}\right)=(0, \operatorname{grad} \phi, 2 X(\phi))
$$

where $X^{j}=g^{0 j}$ is a vector field. As above, if this is $H^{s-1}$, the second component gives that $\phi \in H^{s}$. The domain of $\bar{X} \supset H^{s}$ so the domain of this block is again $H^{s}$.

Now let $u(t, x)$ be the integral curve of our system as determined by Theorem 1.7 which has, by assumption, initial data in the domain of $A$. Hence we get a solution $u(t, x)=\left(\begin{array}{c}\psi \\ \psi, j \\ \psi, 0\end{array}\right) \in D$. Therefore $\psi, 0$ is $C^{1}$; the equation $g^{i j} \frac{\partial \psi, j}{\partial t}=g^{i j} \frac{\partial}{\partial x^{j}} \psi, 0$ together with non-singularity of $g^{i j}$ and the initial data shows $\psi, j=\partial \psi / \partial x^{j}$ and $\psi, 0=\partial \psi / \partial t$. Now the condition $\psi_{, j} \in G^{s}$ becomes

$$
g^{i j} \frac{\partial^{2} \psi}{\partial x^{i} \partial x^{j}} \in H^{s-1}
$$

But $g^{i j}$ is uniformly positive definite, so the standard elliptic theory (cf. Nirenberg [35]) gives $\psi \in H^{s+1}$. Thus we get the result.

It might be noted that this result of having solutions more differentiable than the coefficients is not a general property of first order systems. 
For example, for a scalar equation

$$
\frac{\partial \phi}{\partial t}=\Sigma X^{i} \partial \phi / \partial x^{l}
$$

the solution can, in general, only be $H^{s}$ if the $X^{i}$ are $H^{s}$ (see Appendix 2).

\section{§ 2. Quasi-Linear Symmetric Hyperbolic Systems}

The essential techniques for dealing with quasi-linear systems are rather old, going back to Schauder, Frankl, Petrovsky, and Sobolev. See Courant-Hilbert [15] for a fairly complete bibliography.

Our purpose here is to present a version of this theory which pays particular attention to the differentiability of the coefficients. This care in dealing with the coefficients is necessitated by the applications.

There are several possible sets of hypotheses. The simplest of these was discussed in [21]. Here we wish to treat the case in which an $A^{0}$ term is present. It is not generally appreciated that in the quasi-linear case, this is a non-trivial generalization because the reduction to $A^{0}=\mathrm{Id}$ is no longer possible without destroying some differentiability of the $B$-term. To deal with this situation it is necessary to work with domains of $A^{i}$ as was done in $\S 1$. Otherwise one is confronted with an unnecessary derivative loss ( $H^{s}$ initial data would yield only an $H^{s-1}$ solution). Furthermore, we shall make a few additional special assumptions designed for later use. They are inserted so that we can obtain the sharpest possible bound on $s$, namely $s>n / 2+1$. If one is willing to take larger $s$, the assumptions can be relaxed (see below).

So we are considering an equation of the form

$$
A^{0}(t, x, u) \frac{\partial u}{\partial t}=\Sigma A^{i}(t, x, u) \frac{\partial u}{\partial x^{i}}+B(t, x, u) .
$$

In applications, $A^{0}, A^{i}, B$ will be rational functions of $u$ with non-zero denominator. For purposes of generality we shall just state the relevant properties these functions need. (To verify the conditions for more general classes of functions than rational, one could use Sobolev's "condition T", [40], p. 217.)

For Eq. (1), our assumptions on the coefficients which we shall refer to as assumptions $\left(\tilde{A}_{2}\right)$ are as follows:

(i) $s>n / 2+1$;

(ii) we are given a closed subspace $F^{s-1} C H^{s-1}$ with $F^{s}=F^{s-1} \cap H^{s}$ and an open set $U^{s-1} C F^{s-1}$;

(iii) for $u \in U^{s-1}, A^{0}(t, x, u), A^{i}(t, x, u)$ are $H^{s}$ functions, $B(t, x, u)$ is $H^{s-1}$. 
Remark. This assumption (iii) is the special one referred to above. If $s>n / 2+2$ we could take $u \in U^{s}$. (This is pointed out in the proof below.) The boundedness assumptions follow.

(iv) The maps $u \in U^{s-1} \mapsto A^{0}(t, x, u) \in H^{s}, u \in U^{s-1} \mapsto A^{i}(t, x, u) \in H^{s}$, $u \in U^{s-1} \mapsto B(t, x, u) \in H^{s-1}$ are bounded (map bounded sets to bounded sets) and are $C^{1}$ maps with bounded derivatives.

(v) For $u \in U^{s-1}, A^{0}, A^{i}, B, C$ satisfy the conditions $\left(\mathrm{A}_{2}\right)$ of $\S 1$. The corresponding solutions are assumed to map $F^{s-1}$ to $F^{s-1}$.

(vi) For $v \in U^{s-1}, t \in \boldsymbol{R}$, let the domain of the closure of the operator

$$
A_{v, t} u=\Sigma A^{i}(t, x, v) \partial u / \partial x^{i}: H^{s} \rightarrow H^{s-1}
$$

be denoted $D_{v, t}^{s}$ and let $\|\cdot \cdot\|_{s, v, t}$ denote a Banach space norm on $D_{v, t}^{s}$. Assume that $F^{s-1} \cap D_{v, t}^{s}$ is independent of $t$. Denote the space $F^{s-1} \cap D_{v, t}^{s}$ simply by $D^{s}$ with norm $\|u\| \|_{s}$. Thus for all $u \in D^{s}$ we may suppose

$$
K^{-1}\left[\|u\|_{s-1}+\left\|A_{v, t} u\right\|_{s-1}\right] \leqq\|u\|_{s} \leqq K\left[\|u\|_{s-1}+\left\|A_{v, t} u\right\|_{s-1}\right]
$$

where $K$ is independent of $v \in U^{s-1}, t \in[-T, T], u \in F^{s-1}$.

Finally, we require a special "smoothness" type assumption on $A^{i}$ related to its domain. We state explicitly what we need: form

(vii) For $w_{1}, w_{2} \in U^{s-1}, v \in D^{s}$, we have a Lipschitz estimate of the

$$
\left\|A^{i}\left(t, x, w_{1}\right) \frac{\partial v}{\partial x^{i}}-A^{i}\left(t, x, w_{2}\right) \frac{\partial v}{\partial x^{i}}\right\|_{s-1} \leqq \text { const }\left\|w_{1}-w_{2}\right\|_{s-1}\|v\|_{s}
$$

where the constant may depend on $\left\|w_{1}\right\|_{s-1},\left\|w_{2}\right\|_{s-1}$.

The main result of this section is the following (the asymptotic case is done below).

2.1. Theorem. Let conditions $\left(\tilde{A}_{2}\right)$ hold. Then for $u_{0} \in U^{s-1} \cap D^{s}$, there is an $\varepsilon>0$ and a unique solution $u_{t} \in U^{s-1} \cap D^{s},-\varepsilon<t<\varepsilon$ of (1) which is a $C^{1}$ curve in $H^{s-1}$, and a $C^{0}$ curve in $D^{s}$, and equals $u_{0}$ at $t=0$. Moreover $\varepsilon>0$ can be chosen uniformly in a $D^{s}$ neighborhood of $u_{0}$ and $u_{t}$ varies continuously in $D^{s}$ as a function of $u_{0}$ and $t \in(-\varepsilon, \varepsilon)$.

Proof. For $\delta, M_{1}, M_{2}>0$ to be specified below, let $E$ denote the set of continuous curves

$$
w:[-\delta, \delta] \rightarrow D_{1}^{s}, \quad w(0)=u_{0}
$$

which are also $C^{1}$ curves in $U^{s-1}$ and which satisfy $\|w(t)\|_{s} \leqq M_{1}$ and $\left\|w^{\prime}(t)\right\|_{s-1} \leqq M_{2}$.

We shall set up a contraction $f$ on $E$ in a suitable metric and use the fixed point obtained on the completion of $E$ to get the solution. 
Step 1. Construction of the map $f$.

For $w \in E$ we let $v=f(w)$ be the solution to the system

$$
\left.\begin{array}{rl}
v(0) & =u_{0} \\
A^{0}(t, x, w(t, x)) \frac{\partial v}{\partial t} & =\Sigma A^{i}(t, x, w(t, x)) \frac{\partial v}{\partial x^{i}}+B(t, x, w(t, x))
\end{array}\right\}
$$

Because of (iv) and (v) we see that these coefficients $A^{0}(t, x, w(t, x))$, $A^{i}(t, x, w(t, x)), B(t, x, w(t, x))$ satisfy the conditions $\left(\mathrm{A}_{2}\right)$ of $\S 1$, so we do have a unique solution $v(t) \in D^{s}$. It is $C^{0}$ in $D^{s}$ and $C^{1}$ in $H^{s-1}$.

Step 2. $f$ maps $E$ to $E$ for suitable $\delta, M_{1}, M_{2}$.

From the linear theory in $\S 1$, we know that the Eq. (2) defines a semigroup on $H^{s-1}$ or on $D^{s}$. (As in $\S 1$, the $A^{0}$ term may be grouped with the $H^{s-1} B$ term.) Now we also know from the energy estimates in $\S 1$ that for each fixed $t$, Eq. (2) defines a quasi-contraction in $H^{s-1}$ (that is, the norm of the solution grows exponentially). We can, as in $\S 1$, show that this same property is inherited by the domain $D^{s}$. Thus, using (vi) we conclude that

$$
\|v(t)\|_{s} \leqq \alpha e^{\beta|t|}\left\|u_{0} \mid\right\|_{s}, \quad-\delta \leqq t \leqq \delta
$$

where $\alpha$ is a universal constant (as in the remarks following the assumption (vi)) and $\beta$ depends on $\|w\|_{s-1}$. Thus we may conclude that for $M_{1}$ chosen sufficiently large, we will have $\|v(t)\|_{s} \leqq M_{1}$, if $\delta$ is sufficiently small. From this estimate and the differential equation itself (with $A^{0}$ as a $B$ term) we see that for $M_{2}$ large, the condition $\left\|v^{\prime}(t)\right\|_{s-1} \leqq M_{2}$ will hold. Finally, from $\left\|v^{\prime}(t)\right\|_{s-1} \leqq M_{2}$, it follows from the mean value theorem that $v(t) \in U^{s-1}$ for $\delta$ sufficiently small. This completes step 2 . Note that for $\delta$ small we also may assume that $E$ lies in an $s-1$ ball contained in $U^{s-1}$ centered at $u_{0}$.

Step 3. $f$ is a contraction in the $H^{s-1}$ norm on $E$ for $\delta$ sufficiently small.

For $w \in E$ defined above, let

$$
|w|_{s-1}=\sup _{-\delta \leqq t \leqq \delta}\|w(t)\|_{s-1}
$$

We shall show that $f: E \rightarrow E$ is a (strict) contraction for $\delta$ small; that is,

where $0<k<1$.

$$
\left|f\left(w_{1}\right)-f\left(w_{2}\right)\right|_{s-1} \leqq k\left|w_{1}-w_{2}\right|_{s-1}
$$

In making this estimate we may assume $A^{0}$ is the identity because our assumptions allow us to do this in system 2 without changing the hypotheses on $B$. (Of course this is possible only because we allow $B$ to be $H^{s-1}$ and consequently are working on $D^{s}$ rather than on $H^{s}$. This procedure does not simplify if $A^{0}$ depends only on $u(t, x)$ and not on $t, x$ explicitly.) 
We have from (2),

$$
\begin{aligned}
\frac{d}{d t} \| f\left(w_{1}\right) & -f\left(w_{2}\right) \|_{s-1}^{2} \\
= & \left\langle A^{i}\left(t, x, w_{1}\right) \frac{\partial f\left(w_{1}\right)}{\partial x^{i}}-A^{i}\left(t, x, w_{2}\right) \frac{\partial f\left(w_{2}\right)}{\partial x^{i}}+B\left(t, x, w_{1}\right)\right. \\
& -B\left(t, x, w_{2}\right), f\left(w_{1}-f\left(w_{2}\right)\right\rangle_{s-1} \\
= & \left\langle A^{i}\left(t, x, w_{1}\right) \frac{\partial}{\partial x^{i}}\left[f\left(w_{1}\right)-f\left(w_{2}\right)\right], f\left(w_{1}\right)-f\left(w_{2}\right)\right\rangle_{s-1} \\
& +\left\langle A^{i}\left(t, x, w_{1}\right) \frac{\partial}{\partial x^{i}} f\left(w_{2}\right)\right. \\
& \left.-A^{i}\left(t, x, w_{2}\right) \frac{\partial}{\partial x^{i}} f\left(w_{2}\right), f\left(w_{1}\right)-f\left(w_{2}\right)\right\rangle_{s-1} \\
& +\left\langle\left[B\left(t, x, w_{1}\right)-B\left(t, x, w_{2}\right)\right], f\left(w_{1}\right)-f\left(w_{2}\right)\right\rangle_{s-1} .
\end{aligned}
$$

The first term is estimated by the usual energy inequalities of $\S 1$. It is bounded by

$$
K_{1}\left\|A^{i}\left(w_{1}\right)\right\|_{s}\left\|f\left(w_{1}\right)-f\left(w_{2}\right)\right\|_{s-1}^{2} \leqq K_{2}\left\|f\left(w_{1}\right)-f\left(w_{2}\right)\right\|_{s-1}^{2}
$$

since $\left\|w_{1}\right\|_{s-1}$ is bounded and $w_{1} \mapsto A^{i}\left(w_{1}\right)$ is bounded by assumption. For the second term we use hypothesis (vii). For the third term we have a bound $K_{3}\left\|f\left(w_{1}\right)-f\left(w_{2}\right)\right\|_{s-1}^{2}$ and for the fourth term a bound $K_{4}\left\|w_{1}-w_{2}\right\|_{s-1}\left\|f\left(w_{1}\right)-f\left(w_{2}\right)\right\|_{s-1}$ in virtue of smoothness of $w \mapsto B(t, x, w)$ and the boundedness of its derivative.

Remark. If $s$ were larger we could use a priori bounds in $H^{s-1}$ to get a contraction in $H^{s-2}$ (and a fixed point in $H^{s-1-\varepsilon}$ ), thereby weakening the assumption (ii) to $u \in U^{s-1}$ implies $A^{0}, A^{i}$ are $H^{s-1}$.

Thus we have the estimate

$$
\frac{d}{d t}\left\|f\left(w_{1}\right)-f\left(w_{2}\right)\right\|_{s-1} \leqq C_{1}\left\|f\left(w_{1}\right)-f\left(w_{2}\right)\right\|_{s-1}+C_{2}\left\|w_{1}-w_{2}\right\|_{s-1} .
$$

Thus (by Gronwall's inequality for example) we have

$$
\left\|f\left(w_{1}\right)-f\left(w_{2}\right)\right\|_{s-1} \leqq \frac{C_{2}}{C_{1}}\left\|w_{1}-w_{2}\right\|_{s-1}\left(e^{C_{1} t}-1\right) .
$$

Hence letting $k=\frac{C_{2}}{C_{1}}\left(e^{C_{1} \delta}-1\right)$ we see that if we choose $\delta$ sufficiently small (so $k<1$ ), we get a contraction. 
Thus $f$ extends to a contraction on the completion of $E$ and hence has a fixed point on this completion.

Step 4. The fixed point so obtained represents the solution desired.

Let $u(t)$ be the unique fixed point of $\bar{f}$, the extension of $f$ to the completion $\bar{E}$. Since, as in Step 2, we have $\bar{E} \subset U^{s-1}$, with fixed point $u(t)$ obtained, we do get, as before, a solution $\tilde{u}(t) \in D^{s}$ of the system (2). We assert $\tilde{u}(t)=u(t)$. To show this we want to take the limit of solutions of system (2) (with $A^{0}$ incorporated in $B$ at each step). This is slightly delicate because we are only assuming $s>n / 2+1$. However, it may be achieved by the Trotter-Kato theorem (cf. Yosida [43] and Chernoff [12]) which also holds for time-dependent systems (Kato [28]). The version we need is as follows: if $\frac{\partial u}{\partial t}=X_{\lambda}(t) u$ is a (time-dependent) system depending on a parameter $\lambda$ (in, say, some Banach space) and if $X_{\lambda}(t)$ all have a common core and $X_{\lambda_{n}} \rightarrow X_{\lambda_{0}}$ strongly on this core as $\lambda_{n} \rightarrow \lambda_{0}$, then the solutions converge strongly as well.

In our case, our $\lambda_{n}$ represents successive iterations of a starting point, say $u_{0}$, under $f$. We know they converge strongly in $H^{s-1}$ since $f$ is a contraction. Hence by our assumptions on the coefficients, $X_{\lambda_{n}}$ converges as well. Thus, by the quoted theorem, the iterations converge to $\tilde{u}(t)$ and hence $\tilde{u}(t)=u(t)$.

Remark. See [21] for a more direct proof in case $s$ is larger.

Continuity of the solution with respect to the initial conditions in $H^{s-1}$ will follow at once from the following elementary lemma.

2.2. Lemma. Let $M$ be a complete metric space, $N$ a metric space and $f: M \times N \rightarrow M$ be separately continuous and be such that for each $y \in N$, $f_{y}(x)=f(x, y)$ is a contraction on $M$ with $d\left(f_{y}\left(x_{1}\right), f_{y}\left(x_{2}\right)\right) \leqq k d\left(x_{1}, x_{2}\right)$ for a fixed constant $0<k<1$ independent of $y$. Let $x(y)$ be the unique fixed point of $f_{y}$. Then $y \mapsto x(y)$ is continuous.

Proof. Pick $x_{0} \in M$. Then $x(y)=\operatorname{limit}_{n \rightarrow \infty} f_{y}^{n}\left(x_{0}\right)$ the limit of a sequence of continuous functions. But since $k$ is uniformly less than 1 independent of $y \in N$, the convergence is uniform.

We define $f$, in our case, to be the solution of system (2) on $M=\bar{E}$ with $y=u_{0}$ the initial condition. Lemma 2.2 applies; we just have to check continuity of $f$ separately in $w$ and $y=u_{0}$. But this is clear in $u_{0}$ as (2) is a linear system, and it is clear in $w$ because we have a contraction in that variable.

To obtain continuity in the $D^{s}$ topology requires a bit more care. However it may be proved as follows. Let $u_{n} \rightarrow u_{0}$ be a sequence of initial conditions converging in $D^{s}$, and $u_{n}(t)$ the corresponding solutions. We 
know from above that $u_{n}(t) \rightarrow u(t)$ in $H^{s-1}$. However, as above, from the Trotter-Kato theorem we know that this implies that the corresponding linear semi-groups $F_{t}^{n}$ converge strongly and are equi-continuous on $D^{s}$. Letting $F_{t}$ be the limiting solution, we have

$$
\begin{aligned}
\left\|F_{t}\left(u_{0}\right)-F_{t}\left(u_{n}\right)\right\| \|_{s} \leqq & \left\|F_{t}\left(u_{0}\right)-F_{t}^{N}\left(u_{0}\right)\right\|_{s}+\left\|F_{t}^{N}\left(u_{0}\right)-F_{t}^{N}\left(u_{n}\right)\right\|_{s} \\
& +\left\|F_{t}^{N}\left(u_{n}\right)-F_{t}\left(u_{n}\right)\right\|_{s} \\
\leqq & \left\|F_{t}\left(u_{0}\right)-F_{t}^{N}\left(u_{0}\right)\right\|_{s}+(\text { const })\left\|u_{0}-u_{n}\right\| \|_{s} \\
& +\left\|F_{t}^{N}\left(u_{n}\right)-F_{t}\left(u_{n}\right)\right\|_{s} .
\end{aligned}
$$

Given $\varepsilon>0$ choose $n \geqq n_{1}$ so the middle term is $<\varepsilon / 3$, then choose $N$ so large that the first and last terms are $<\varepsilon / 3$ (which is possible by strong convergence). Hence we get continuity of $F_{t}\left(u_{0}\right)$ in $u_{0}$.

As in $\S 1$ we may also consider the situation in which our coefficients and unknowns satisfy a certain asymptotic condition. Our assumptions are as follows, which we refer to as $\left(\tilde{A}_{3}\right)$.

(i) $s>n / 2+1$.

(ii) For $w \in C_{b}^{s}$ we have a neighborhood $U^{s-1}$ of $w$ in a closed subspace $F^{s-1}$ of $H_{w}^{s-1}$ (defined in $\S 1$ ). Also, we have fixed $\tilde{A}_{t}^{0}, \tilde{A}_{t}^{i}, \tilde{B}_{t} \in C_{b}^{s}$ such that $A_{t}^{0}, A_{t}^{i}, B_{t}$ are asymptotic to them for each $u \in U^{s}$ and in these asymptotic spaces the conditions $\left(\mathrm{A}_{3}\right)$ of $\S 1$ hold.

(iii) The conditions $\left(\tilde{A}_{2}\right)$ above hold with the appropriate asymptotic conditions imposed.

2.3. Theorem. Under these conditions, the conclusions of Theorem 2.1 remain valid with the appropriate asymptotic conditions, i.e., for $u_{0} \in U^{s-1}$ $\cap\left(D^{s}+w\right)$ we have a unique solution of (1) in the same space, and the solution varies continuously with the initial data.

Proof. Argue as in Theorem 1.7 making use of 1.6. and 2.1.

This concludes the basic existence theory. Now we shall give a few properties of the solutions. In the applications these properties will give further information about the Einstein system.

2.4. Theorem. Let $\tilde{A}_{3}$ (or $\tilde{A}_{2}$ ) hold and let $\mathscr{D}_{\mathrm{s}} \subset \boldsymbol{R} \times\left(D^{s}+w\right)$ be the domain of the maximally extended solutions for the system (1). Then

(a) $\mathscr{D}_{s}$ is open

(b) integral curves of (1) are unique on their domains of maximal extension.

Proof. This follows from the continuity of the solution with respect to the initial data in the same way as for ordinary differential equations; cf. Lang [30]. 
Thus for $(t, u) \in \mathscr{D}_{\mathrm{s}}$ we have $F_{t}(u)$ defined as the solution to (1) with initial condition $u$. Then $F_{t}$ maps an open subset of $D^{s}$ to $D^{s}$. Globally, we have $F: \mathscr{D}_{s} \rightarrow D^{s}$ a continuous map.

2.5. Corollary. Suppose $F_{t}\left(u_{0}\right)$ exists in $D^{s}$ for all $t \geqq 0$. Let $T>0$. Then there is a neighborhood of $u_{0}$ in which solutions exist for a time $t \geqq T$.

Proof. Immediate from openness of $\mathscr{D}_{s}$.

For second order systems this result was proven (in an apparently more complicated way) by Choquet-Bruhat [10].

Sometimes this result is referred to as "stability" of $u_{0}$, but that is misleading; the usual notion of stability is a "second order" property implying that near $u_{0}$ solutions will be defined for all $t \geqq 0$ and will remain close to the solution through $u_{0}$. On the other hand, 2.5 merely expresses continuity in the initial data. A less obvious result is the following (compare also Choquet-Bruhat [10]):

2.6. Theorem. Let $\tilde{A}_{2}$ or $\tilde{A}_{3}$ hold and $F: \mathscr{D}_{s} \rightarrow D^{s}$ be as above. Then for fixed $t, F_{t}: D^{s} \rightarrow D^{s}$ (locally defined) is a $C^{\infty}$ mapping.

This property of being smooth for $t$ fixed is actually quite general. A general theorem, of which 2.6 is a special case, is given in Appendix A.

2.7. Theorem.(Regularity). Let $F_{t}$ be as above and $u_{0} \in D^{s+1}, s>n / 2+3$. Then $F_{t}\left(u_{0}\right)$ is in $D^{s+1}$ as long as it is defined in $D^{s}$. In particular the time of existence is independent of $s$. In other words for $r>s$, possibly $r=\infty$, we have

$$
\mathscr{D}_{r}=\left\{(t, u) \in \mathscr{D}_{s} \mid u \in D^{r}\right\} .
$$

Proof. The trick is to look at the differential equation satisfied by the second spatial derivative of $u$, the solution found in $D^{s} C H^{s-1}$. Now

$$
A^{0}(t, x, u) \frac{\partial u}{\partial t}=A^{i}(t, x, u) \frac{\partial u}{\partial x^{i}}+B(t, x, u)
$$

so if $D u$ is the first differential of $u$ with respect to $x$,

$$
\begin{aligned}
A^{0}(t, x, u) \frac{\partial}{\partial t}(D u)= & D_{2} A^{i}(t, x, u) \frac{\partial u}{\partial x^{i}}+D_{3} A^{i}(t, x, u) \cdot D u \cdot \frac{\partial u}{\partial x^{i}} \\
& +A^{i}(t, x, u) \frac{\partial}{\partial x^{i}} D u+D B(t, x, u)-\left[D A^{0}(t, x, u)\right] \frac{\partial u}{\partial t}
\end{aligned}
$$

where $D_{2} A^{i}$ and $D_{3} A^{i}$ are the partial derivatives with respect to the second and third variables, respectively. If we consider this as a linear equation in the unknown $v=D u$ of the form

$$
\frac{\partial v}{\partial t}=A^{i} \frac{\partial v}{\partial x^{i}}+C \cdot v+D
$$


then we must treat $D_{3} A^{i}(t, x, u) \frac{\partial u}{\partial x^{i}}$ as a coefficient. However, since $u$ is only $H^{s-1}, \frac{\partial u}{\partial x^{i}}$ is only $H^{s-2}$. However, if we differentiate (2), it is easy to see that $w=D^{2} u$ satisfies

$$
A^{0} \frac{\partial w}{\partial t}=A^{i} \frac{\partial w}{\partial x^{i}}+\tilde{C} \cdot w+\tilde{D}
$$

where now $A^{i}, \tilde{C}, \tilde{D}$ are $H^{s-2}$ functions, $\frac{\partial D u}{\partial x^{i}}$ is incorporated into $w$ and we substitute from (1) for $\frac{\partial u}{\partial t}$. The reason is just that second derivatives do not occur multiplied together as the first ones did. Hence, since $s>n / 2+3$, the coefficients are in $H^{r}, r=s-2>n / 2+1$, with $A^{i}$ in $H^{s-1}$ so by the linear theory $w$, which is initially in $D^{s-1}$, remains in $D^{s-1}$. Hence $u$ remains in $D^{s+1}$.

We shall apply this regularity result to the Einstein system to show that $C^{\infty}$ Cauchy data remains $C^{\infty}$.

\section{§ 3. Existence Theorem for the Exterior Einstein Equations}

In this section we wish to show how the classical existence theorems of Lichnerowicz and Choquet-Bruhat $[3,33]$ (including the improvement recently obtained using a result of Dionne [16]) as well as some recent modifications $[6,7]$ can all be obtained from the symmetric hyperbolic theory above. Since our improvements of the results are technical ones, we shall not include all the details.

We point out that the second order theory of Leray used by the above authors requires strict hyperbolicity of the equations. Sometimes when strict hyperbolicity fails, the system may still be put in the form of a symmetric hyperbolic system; cf. Friedrichs [24]. We also point out that the symmetric hyperbolic theory admits a global intrinsic treatment on manifolds; this is the subject of Part II (see also [34]).

Some of the technically delicate points we deal with follow: a direct treatment of the asymptotic conditions and globally defined "coordinate transformations" $H^{s}$ asymptotic to the identity; an improvement (from $H^{s}$ to $H^{s+1}$ ) of the degree of differentiability of the coordinate transformation relating two solutions of the equations $R_{\mu \nu}=0$ with the same Cauchy data; existence of solutions in the class $\mathrm{H}^{4}$; the latter is given in $[6,8]$. Moreover, we also show that the solutions to the Cauchy problem depend continuously on the initial data in the $H^{s}$ topology and that $C^{\infty}$ initial data give $C^{\infty}$ solutions; cf. $[7,9,10,25]$. Finally we show that the time $T$ solutions are $C^{\infty}$ functions of the initial data. 
Details not given below for the most part can be routinely supplied. We now summarize our notations.

Greek indices run from 0 to 3 , latin indices from 1 to 3 . Local coordinates are written $x^{\alpha}=\left(t, x^{i}\right)=\left(x^{0}, x^{i}\right)$. We take $c=1$ and our Lorentz metrics $g_{\mu v}$ have signature $(-,+,+,+)$. The summation convention is employed.

Christoffel Symbols:

$$
\Gamma_{\alpha \beta}^{\mu}=\frac{1}{2} g^{\mu \lambda}\left[\frac{\partial g_{\lambda \alpha}}{\partial x^{\beta}}+\frac{\partial g_{\lambda \beta}}{\partial x^{\alpha}}-\frac{\partial g_{\alpha \beta}}{\partial x^{\hat{\lambda}}}\right] .
$$

Contracted Christoffel Symbols:

$$
\begin{aligned}
\Gamma^{\mu} & =g^{\alpha \beta} \Gamma_{\alpha \beta}^{\mu} \\
& =-\frac{1}{V-\operatorname{det} g} \frac{\partial}{\partial x^{\nu}}\left(\sqrt{-\operatorname{det} g} g^{\nu \mu}\right)
\end{aligned}
$$

where $\operatorname{det} g=$ determinant of $g_{\mu \nu}$.

Riemann Curvature Tensor:

$$
\begin{aligned}
R_{\alpha \mu \beta \nu}= & \frac{1}{2}\left[\frac{\partial^{2} g_{\mu \beta}}{\partial x^{v} \partial x^{\alpha}}+\frac{\partial^{2} g_{\nu \alpha}}{\partial x^{\mu} \partial x^{\beta}}-\frac{\partial^{2} g_{\mu \nu}}{\partial x^{\alpha} \partial x^{\beta}}-\frac{\partial^{2} g_{\alpha \beta}}{\partial x^{\mu} \partial x^{v}}\right] \\
& -g_{\varrho \sigma} \Gamma_{\mu \nu}^{\varrho} \Gamma_{\alpha \beta}^{\sigma}+g_{\varrho \sigma} \Gamma_{\mu \alpha}^{\varrho} \Gamma_{v \beta}^{\sigma} .
\end{aligned}
$$

Ricci Curvature Tensor:

$$
\begin{aligned}
R_{\mu v}= & g^{\alpha \beta} R_{\alpha \mu \beta v} \\
= & \frac{1}{2} g^{\alpha \beta}\left[-\frac{\partial^{2} g_{\mu v}}{\partial x^{\alpha} \partial x^{\beta}}-\frac{\partial^{2} g_{\alpha \beta}}{\partial x^{\mu} \partial x^{v}}+\frac{\partial^{2} g_{\mu \beta}}{\partial x^{\alpha} \partial x^{v}}+\frac{\partial^{2} g_{v \alpha}}{\partial x^{\beta} \partial x^{\mu}}\right] \\
& -g_{\varrho \sigma} \Gamma_{\mu v}^{\varrho} \Gamma^{\sigma}+g^{\alpha \beta} g_{\varrho \sigma} \Gamma_{\mu \alpha}^{\varrho} \Gamma_{v \beta}^{\sigma} \\
= & \frac{\partial \Gamma_{\mu v}^{\alpha}}{\partial x^{\alpha}}-\frac{\partial \Gamma_{\alpha \mu}^{\alpha}}{\partial x^{v}}+\Gamma_{\mu \nu}^{\alpha} \Gamma_{\alpha \beta}^{\beta}-\Gamma_{\mu \beta}^{\alpha} \Gamma_{v \alpha}^{\beta} .
\end{aligned}
$$

Scalar Curvature:

Einstein Tensor:

$$
R=g^{\alpha \beta} R_{\alpha \beta} .
$$

$$
G_{\mu \nu}=R_{\mu \nu}-\frac{1}{2} g_{\mu \nu} R .
$$

Laplace-Beltrami Operator on Scalars:

$$
\begin{aligned}
\square \phi & =-\frac{1}{\sqrt{-\operatorname{det} g}} \frac{\partial}{\partial x^{\beta}}\left[\sqrt{-\operatorname{det} g} g^{\beta \alpha} \frac{\partial \phi}{\partial x^{\alpha}}\right] \\
& =-g^{\alpha \beta} \frac{\partial^{2} \phi}{\partial x^{\alpha} \partial x^{\beta}}+\Gamma^{\alpha} \frac{\partial \phi}{\partial x^{\alpha}} .
\end{aligned}
$$

The covariant derivative is denoted $t^{\alpha \beta \ldots}{ }_{\mu \nu \ldots, \sigma \sigma}$ etc. 
The Cauchy Problem is as follows:

(Existence). Let $\stackrel{\circ}{\mu \nu}_{\mu \nu}\left(x^{i}\right)$ be an $H^{s}$ asymptotically flat metric (see below), $s \geqq 4$, and $\stackrel{\circ}{k \nu}_{\nu}\left(x^{i}\right)$ an $H^{s-1}$ symmetric tensor (asymptotic to zero). Assume $\dot{\sigma}_{\mu}^{0}\left(x^{i}\right)=0$. Find $\varepsilon>0$ and an $H^{s}$ asymptotically flat spacetime $g_{\mu \nu}\left(t, x^{l}\right),-\varepsilon<t<\varepsilon$ such that $g_{\mu \nu}\left(0, x^{i}\right)=\stackrel{\circ}{g}_{\mu \nu}\left(x^{i}\right), \frac{\partial g_{\mu \nu}}{\partial t}\left(0, x^{i}\right)=\dot{\circ}_{\mu \nu}\left(x^{i}\right)$ and $R_{\mu v}=0$.

(Uniqueness). Let $g_{\mu \nu}$ and $h_{\mu \nu}$ be two $H^{s}$ asymptotically flat spacetimes with the same Cauchy data and which satisfy $R_{\mu v}=0$. Find an $\varepsilon^{\prime}$ and an $H^{s+1}$ coordinate transformation $\bar{x}^{\mu}\left(x^{\alpha}\right)$ globally defined in space and spatially asymptotic to the identity such that

$$
h_{\mu \nu}\left(\bar{x}^{\lambda}\right)=\frac{\partial x^{\alpha}}{\partial \bar{x}^{\mu}}\left(\bar{x}^{\lambda}\right) \frac{\partial x^{\beta}}{\partial \bar{x}^{\nu}}\left(\bar{x}^{\lambda}\right) g_{\alpha \beta}\left(x^{\varrho}\left(\bar{x}^{\lambda}\right)\right) \text {. }
$$

Both of these problems as well as related ones will be answered affirmatively.

By asymptotically flat, we mean that $\stackrel{\circ}{g}_{\mu v}$ is $H^{s}$ asymptotic to some given comparison metric. In practice this means $\stackrel{\circ}{g}_{\mu \nu}$ is $H^{s}$ asymptotic to an asymptotically Schwarschild type metric $\tilde{g}_{\mu v}$, or $\stackrel{\circ}{g}_{\mu \nu}-\tilde{g}_{\mu \nu} \in H^{s}$. Here our comparison metric $\tilde{g}_{\mu \nu}$ looks like $\mathbf{1}_{m}=\eta_{\mu \nu}+\frac{2 m}{r} \delta_{\mu \nu}$ for $r$ sufficiently large, where $m \geqq 0$ is a constant and $\eta_{\mu \nu}$ is the Minkowski matrix (see $1.7,1.8,2.3)$. The existence theory then guarantees that these asymptotic conditions will automatically be maintained in time.

As with the second order theory we make use of harmonic coordinates defined by the condition

$$
\Gamma^{\mu}=g^{\alpha \beta} \Gamma_{\alpha \beta}^{\mu}=0 .
$$

In this case, the system $R_{\mu \nu}=0$ simplifies considerably.

An algebraic computation (see, e.g., Fock [22], p. 423) shows that

where

$$
R_{\mu \nu}=R_{\mu \nu}^{(h)}+\frac{1}{2}\left(g_{\mu \nu} \frac{\partial \Gamma^{\alpha}}{\partial x^{v}}+g_{v \alpha} \frac{\partial \Gamma^{\alpha}}{\partial x^{\mu}}\right)
$$

and

$$
R_{\mu \nu}^{(h)}=-\frac{1}{2} g^{\alpha \beta} \frac{\partial^{2} g_{\mu \nu}}{\partial x^{\alpha} \partial x^{\beta}}+H_{\mu \nu}\left(g_{\alpha \beta}, \frac{\partial g_{\alpha \beta}}{\partial x^{\lambda}}\right)
$$

$$
\begin{aligned}
& H_{\mu \nu}\left(g_{\alpha \beta}, \frac{\partial g_{\alpha \beta}}{\partial x^{\lambda}}\right)=g^{\alpha \beta} g_{\varrho \sigma} \Gamma_{\mu \beta}^{\varrho} \Gamma_{\nu \alpha}^{\sigma} \\
& \quad+\frac{1}{2}\left(\frac{\partial g_{\mu \nu}}{\partial x^{\alpha}} \Gamma^{\alpha}+g_{\nu \lambda} \Gamma_{\alpha \beta}^{\lambda} g^{\alpha \gamma} g^{\beta \delta} \frac{\partial g_{\gamma \delta}}{\partial x^{\mu}}+g_{\mu \lambda} \Gamma_{\alpha \beta}^{\lambda} g^{\alpha \gamma} g^{\beta \delta} \frac{\partial g_{\gamma \delta}}{\partial x^{v}}\right)
\end{aligned}
$$


Thus if $\Gamma^{\alpha}=0, R_{\mu \nu}$ reduces to $R_{\mu \nu}^{(h)}$. Note that $H_{\mu \nu}$ is homogeneous quadratic in $\partial g_{\mu \nu} / \partial x^{\alpha}$ and is rational in $g_{\mu v}$ with non-zero dominator $\operatorname{det}\left(g_{\mu v}\right)$.

The principal part of the system $R_{\mu \nu}^{(h)}=0$, namely the operator $-\frac{1}{2} g^{\alpha \beta} \partial^{2} / \partial x^{\alpha} \partial x^{\beta}$, operates the same way on each component $g_{\mu \nu}$ of the system so that the highest order terms are completely uncoupled. Such systems are said to be weakly coupled, and are a particular case of the strictly hyperbolic systems of Leray [32].

From Leray's theorem [32] for strictly hyperbolic second order systems as improved by Dionne [16], Choquet-Bruhat [6, 7] concludes that $H^{s}, H^{s-1}$ Cauchy data for the system $R_{\mu \nu}^{(h)}=0$ evolve into an $H^{s}$ spacetime. In this section we shall prove this result using the theory of first order quasi-linear hyperbolic systems which we developed in $\S 2$. First we shall set up our asymptotic conditions.

Let $T_{2}^{\mathrm{sym}}\left(\boldsymbol{R}^{4}\right)$ denote the 10 dimensional vector space of symmetric 2-covariant tensors on $\boldsymbol{R}^{4}$ (symmetric bilinear forms) and let $\Lambda\left(\boldsymbol{R}^{4}\right)$ denote the open set $C T_{2}^{\text {sym }}$ of forms $g_{\mu \nu}$ with Lorentz signature $(-,+,+,+)$; thus the submatrix $g_{i j}$ is positive-definite and $g_{00}<0$. Let $\mathbf{1}_{m}$ denote the Minkowski matrix $\eta_{\mu \nu}$ plus a term of class $C_{b}^{s}$ which for large $r$ is $\frac{2 m}{r} \delta_{\mu \nu}$ (all we really need is an asymptotic value satisfying the sort of conditions spelled out in $\$ \S 1,2$ ). Thus, as in $1.8, \mathbf{1}_{m}$ is $H^{s}$ asymptotic to a $t=$ const. slice of the Schwarschild metric in isotropic coordinates.

Definition. A map $g: \boldsymbol{R}^{3} \rightarrow \Lambda\left(\boldsymbol{R}^{4}\right)$ is called $H^{s}$ asymptotically flat (with mass $m$ ) if $g-\mathbf{1}_{m} \in H^{s}\left(\boldsymbol{R}^{3}, \Lambda\left(\boldsymbol{R}^{4}\right)\right.$ ).

Of course, here we put a positive-definite metric on $\Lambda\left(\boldsymbol{R}^{4}\right)$ when forming the Sobolev space $H^{s}\left(\boldsymbol{R}^{3}, \Lambda\left(\boldsymbol{R}^{4}\right)\right)$.

Let us fix $m$ and put

and

$$
\mathscr{L}^{s}=\left\{g: \boldsymbol{R}^{3} \rightarrow \Lambda\left(\boldsymbol{R}^{4}\right) \mid g \text { is } H^{s} \text { asymptotically flat }\right\}
$$

$$
\mathscr{K}^{s}=H^{s}\left(\boldsymbol{R}^{3}, T_{2}^{\mathrm{sym}}\left(\boldsymbol{R}^{4}\right)\right) .
$$

The space $\mathscr{L}^{s} \times \mathscr{K}^{s-1}, s>n / 2+2, n=3$, will be the space of Cauchy data for the Einstein equations. Thus for $g \in \mathscr{L}^{s}$, it follows that $g \rightarrow \mathbf{1} C^{2}$ at infinity and for $k \in \mathscr{K}^{s-1}, k \rightarrow 0$ in the $C^{1}$ norm at infinity. (This follows easily from the Sobolev theorems.)

For $\varepsilon>0$, let $I=(-\varepsilon, \varepsilon)$ and $V_{4}=I \times \boldsymbol{R}^{3}$.

Definition. By an $H^{s}$ asymptotically flat Lorentz metric on $V_{4}$, we mean a map $g^{L}: V_{4} \rightarrow \Lambda\left(\boldsymbol{R}^{4}\right)$ such that

(i) for each $t \in I, g^{L}(t, \cdot) \in \mathscr{L}^{s}$ such that $t \mapsto g^{L}(t, \cdot) \in \mathscr{L}^{s}$ is continuous and

(ii) $g^{L}$ is locally jointly $H^{s}$ in $(t, x)$. 
We shall also refer to $g^{L}$ as an $H^{s}$ spacetime.

Note that as part of our assumptions concerning $\Lambda\left(\boldsymbol{R}^{4}\right)$, the surfaces $\left\{t_{0}\right\} \times \boldsymbol{R}^{3} \subset V_{4}$ are space-like for $g^{L}$ (that is, for $v \in \boldsymbol{R}^{3}, v \neq 0, g^{L}\left(t_{0}, x\right)$ $\cdot((0, v),(0, v))>0)$.

From the dynamical point of view we shall see that, alternative to (ii), one may wish to require that $g^{L}$ be a $C^{0}$ curve in $\mathscr{L}^{s}$ and a $C^{1}$ curve in $\mathscr{L}^{s-1}$ (see $\S 2$ and below).

We shall also wish to consider $H^{s}$ coordinate transformations which are asymptotically the identity.

Definition. A map $f: V_{4} \rightarrow \boldsymbol{R}^{4}$ will be called an $H^{s}$ coordinate transformation asymptotic to the identity if the following conditions hold:

(i) $f$ is a local $H^{s}$ diffeomorphism in $(t, x)$,

(ii) $f(0, x)=(0, x), \frac{\partial f}{\partial t}(0, x)=(1,0)$,

(iii) writing $f(t, x)=(\bar{t}(t, x), \bar{x}(t, x)), \bar{x}$ is a $C^{1}$ diffeomorphism of $\boldsymbol{R}^{3}$ to $\boldsymbol{R}^{3}$ for each $t$, with $\bar{x}-x \in H^{s}$ and $t \mapsto \bar{x}(t, \cdot)-x \in H^{s}$ continuous, and

(iv) $\bar{t}$ is an $H^{s}$ diffeomorphism for each fixed $x$ and $\bar{t}-t \in H^{s}\left(\boldsymbol{R}^{3}, \boldsymbol{R}\right)$ for each fixed $t$.

In the sequel, we shall just refer to $f$ as an $H^{s}$ coordinate transformation.

If $g$ is a Lorentz metric on $V_{4}$, and $\phi: V_{4} \rightarrow V_{4}$ a diffeomorphism, we recall that the pull-back of $g$ by $\phi$ is the metric given by

$$
\left(\phi^{*} g\right)(m)\left(X_{m}, Y_{m}\right)=g(\phi(m)) \cdot\left(T_{m} \phi \cdot X_{m}, T_{m} \phi \cdot Y_{m}\right)
$$

where $m \in V_{4}, X_{m}, Y_{m} \in T_{m} V_{4} \approx \boldsymbol{R}^{4}$ and where $T_{m} \phi$ is the tangent (derivative) of $\phi$. The push-forward is $\left(\phi^{-1}\right)^{*} g$. If we write $\bar{g}=\left(\phi^{-1}\right)^{*} g$ and let $\bar{x}^{\alpha}=\phi^{\alpha}\left(x^{\beta}\right)$ then we have the coordinate expression

$$
\bar{g}_{\mu \nu}\left(\bar{x}^{\lambda}\right)=g_{\alpha \beta}\left(x^{\beta}\left(\bar{x}^{\lambda}\right)\right) \frac{\partial x^{\alpha}}{\partial \bar{x}^{\mu}}\left(\bar{x}^{i}\right) \frac{\partial x^{\beta}}{\partial \bar{x}^{v}}\left(\bar{x}^{\lambda}\right) .
$$

We shall prove the following, as it requires some care.

3.1. Lemma. Let $s>n / 2$ and let $g^{L}$ be an $H^{s}$ asymptotically flat Lorentz metric on $V_{4}$ and $f$ an $H^{s+1}$ coordinate transformation (asymptotic to the identity). Then on $\left(-\varepsilon^{\prime}, \varepsilon^{\prime}\right) \times \boldsymbol{R}^{3}$ for some $\varepsilon^{\prime}>0,\left(f^{-1}\right)^{*} g^{L}$ is also on $H^{s}$ asymptotically flat Lorentz metric.

Proof. We let $\mathscr{D}_{I}^{s}\left(\boldsymbol{R}^{n}\right)$ denote the $H^{s}$ diffeomorphisms of $\boldsymbol{R}^{n}$ asymptotic to the identity, as defined above. We shall use the following (Cantor [5]): 
For $s>n / 2+1, \mathscr{D}_{I}^{s}\left(\boldsymbol{R}^{n}\right)$ is a topological group and acts continuously by composition on $H^{s}\left(\boldsymbol{R}^{n}, \boldsymbol{R}^{m}\right)$.

This is a generalization with asymptotic conditions of the known fact that the composition of an $H^{s}$ map with an $H^{s}$ diffeomorphism is again $H^{s}$ (see Sobolev [40], Ebin [18]). Also, products of $H^{s}$ functions are $H^{s}$ (this ring property of $H^{s}$ was used in $\S \S 1,2$ ). From these facts it follows that $\bar{g}=\left(f^{-1}\right)^{*} g$ is locally $H^{s}$, as $\bar{g}$ is formed from compositions and products.

Of course, it is clear formally that $\bar{g} \rightarrow \mathbf{1}_{m}$ at spatial infinity since $f \rightarrow i d$ at spatial infinity.

This may be obtained precisely from the above results as follows. The map $f$ is an $H^{s}$ diffeomorphism of $\{t\} \times \boldsymbol{R}^{3}$ onto $\bar{t} \times \boldsymbol{R}^{3}$ asymptotic to the identity. If we then write out $\bar{g}-\mathbf{1}_{m}$ we see by Cantor's result and by Lemma 1.6 that $\bar{g}-\mathbf{1}_{m}$ is $H^{s}$ and is continuous in $t$ (note that $D f \in H_{\mathrm{Id}}^{\mathrm{s}}$ ). Since $\mathscr{L}^{s}$ is open in $H^{s}$ and $f$ starts out the identity, as does $D f, \bar{g}$ will be in $\mathscr{L}^{s}$ for some $t$-interval $\left(-\varepsilon^{\prime} \varepsilon^{\prime}\right)$.

Let $x^{\lambda}$ be a coordinate system on $V_{4}$, and $g_{\mu v}$ a given spacetime. The coordinate system is called harmonic if $\Gamma^{\mu}\left(x^{\lambda}\right)=0$. This requirement is equivalent to saying that the coordinate functions themselves are harmonic functions: $\square x^{\mu}=0$ (since $\square \phi=-g^{\alpha \beta} \hat{\partial}^{2} \phi / \partial x^{\alpha} \partial x^{\beta}+\Gamma^{\alpha} \hat{\partial} \phi / \partial x^{\alpha}$ ).

In the next section we shall show that an arbitrary $H^{s}$ spacetime can be transformed by an $H^{s+1}$ coordinate transformation to one that satisfies the harmonic condition. This new coordinate system is a global one.

The importance of harmonic coordinates and of the system $R_{\mu \nu}^{(h)}=0$ is that it is sufficient to solve the Cauchy problem for $R_{\mu \nu}^{(h)}=0$. This remarkable fact, discovered by Fourès-Bruhat [3], is based on the observation that the condition $\Gamma^{\mu}=0$ on the Cauchy data is automatically propagated off the $t=0$ hypersurface for solutions of $R_{\mu \nu}^{(h)}=0$, provided that the initial data also satisfies $G_{\mu}^{0}\left(0, x^{t}\right)=0$ where $G_{\mu \nu}=R_{\mu \nu}-\frac{1}{2} g_{\mu \nu} R$ is the Einstein tensor. The latter condition is a postiori necessary since $R_{\mu \nu}=0$ iff $G_{\mu \nu}=0$, and $G_{\mu}^{0}$ can be computed from the Cauchy data $g_{\mu v}\left(0, x^{i}\right), \frac{\partial g_{\mu v}}{\partial t}\left(0, x^{i}\right)$ alone (it is well-known that $G_{\mu}^{0}$ depends only on the first order time derivatives of $g_{\mu \nu}$ ). Similarly $\Gamma^{\mu}$ can be computed from the Cauchy data above. We also remark that in fact $G_{\mu}^{0}$ does not depend on $\partial g_{0 \mu} / \partial t$. For example, in the notation of [20],

$$
-4 G_{0}^{0}=\mathscr{H}=\frac{1}{2}\left((\operatorname{Tr} k)^{2}-k \cdot k\right)+2{ }^{3} R, \quad-2 N G_{i}^{0}=\delta((\operatorname{Tr} k) g-k),
$$

where

$$
k_{i j}=\frac{1}{N}\left(\dot{g}_{i j}+g_{0 i \mid j}+g_{0 j \mid i}\right), \quad N=\left(-g^{00}\right)^{-1 / 2}
$$


which demonstrates the assertion (in the expression for $k_{i j},{ }_{\mid i}$ denotes covariant differentiation with respect to the 3 -metric $g_{i j}$ ).

3.2. Lemma. Let $(\stackrel{\circ}{g}, \stackrel{\circ}{k}) \in\left(\mathscr{L}^{s}, \mathscr{K}^{s-1}\right)$ and suppose $s>3 / 2+2$. Assume $\stackrel{\circ}{\Gamma}^{\mu}\left(x^{i}\right)=0$ and $\stackrel{\circ}{G}_{\mu}^{0}\left(x^{i}\right)=0$ (the superscript refers to the fact that $\Gamma^{\mu}$ and $G_{\mu}^{0}$ are computed from $\stackrel{\circ}{g}, \stackrel{\circ}{k})$. Let $g_{\mu \nu}$ be an $H^{s}$ spacetime satisfying $g_{\mu \nu}\left(0, x^{i}\right)$ $=\stackrel{\circ}{g}_{\mu v}\left(x^{i}\right), \frac{\partial g_{\mu \nu}}{\partial t}\left(0, x^{i}\right)=\stackrel{\circ}{k}_{\mu \nu}\left(x^{i}\right)$ and $R_{\mu \nu}^{(h)}=0$. Then $\Gamma^{\mu}\left(t, x^{i}\right)=0$.

Remarks. The basic computation appears in [3]. However, in the present situation some care is required concerning the amount of differentiability. In particular we are in the delicate situation of dealing with $H^{s}$ for low $s$ in which case $H^{s}$ is no longer a ring (closed under multiplication). The idea is to work out $G^{\mu v}{ }_{\mid v}=0$. Since $g_{\mu v} \in H^{4}, g_{\mu v} \in C_{b}^{2}$, and the third derivative terms occur linearly multiplied by uniformly bounded functions, the computation of $G^{\mu v}{ }_{\mid v}=0$ as if it were $C^{\infty}$ can be justified. One ends up with a system of the form

$$
\frac{1}{2} g^{\beta \nu} \frac{\partial^{2} \Gamma^{\mu}}{\partial x^{\beta} \partial x^{\nu}}+A_{\alpha}^{\beta \mu}\left(g_{\mu \nu}, \frac{\partial g_{\mu \nu}}{\partial x^{2}}\right) \frac{\partial \Gamma^{\alpha}}{\partial x^{\beta}}=0,
$$

where $\partial^{2} \Gamma^{\mu} / \partial x^{\beta} \partial x^{v}$ is an $H^{1}$ function. As was explained in Theorem 1.8, this reduces to a first order linear symmetric hyperbolic system in $\left(\Gamma^{\mu}, \Gamma^{\mu}{ }_{i}, \Gamma^{\mu}{ }_{0}\right) \in H^{2}$. Since the coefficients of (1) are in $C^{1}$, we have uniqueness in the class $H^{1}$, and since the initial data is zero, so is the solution. Thus one can obtain 3.2 even for this low value of $s$.

Thus an $H^{s}$ solution of $R_{\mu \nu}^{(h)}=0$ with prescribed Cauchy data and $\stackrel{\circ}{\Gamma}^{\mu}=0, \stackrel{\circ}{G}_{\mu}^{0}=0$ is also a solution of $R_{\mu \nu}=0$ (since $\Gamma^{\mu}=0$ ). As we shall see in the next theorems, if the $\stackrel{\circ}{\Gamma}^{\mu}$ are not zero, we can make them zero by a suitable coordinate transformation.

Our main new technique is given in the proof of the following:

3.3. Theorem. (Existence). Let $s \geqq 4$ (or $s>3.5$ if not an integer). Let $\left(\stackrel{\circ}{g}_{\mu v}, \stackrel{k}{\mu v}_{\mu \nu}\right) \in \mathscr{L}^{s} \times \mathscr{K}^{s-1}$. Then there exists an $\varepsilon>0$ and a unique $H^{s}$ asymptotically flat Lorentz metric $g^{L}\left(t, x^{i}\right), t \in I=(-\varepsilon, \varepsilon)$ such that $t \mapsto g^{L}(t, \cdot) \in \mathscr{L}^{s}$ is $C^{0}$, is $C^{1}$ into $\mathscr{L}^{s-1}, g^{L}\left(0, x^{i}\right)=\stackrel{g}{g}\left(x^{i}\right), \frac{\partial g^{L}}{\partial t}\left(0, x^{i}\right)=\grave{k}\left(x^{i}\right)$ and $R_{\mu \nu}^{(h)}=0$.

The solution depends continuously on the initial data in $\mathscr{L}^{s} \times \mathscr{K}^{s-1}$, and moreover, for $T$ fixed, $|T|<\varepsilon$, the solution is a $C^{\infty}$ function of the initial data. If the initial data is $C^{\infty}$, so is the solution.

Case A. If the Cauchy data satisfy $\stackrel{G}{\mu}_{\mu}^{0}=0, \stackrel{\circ}{\Gamma}^{\mu}=0$, then $g_{\mu \nu}$ so obtained satisfies $R_{\mu v}=0$. 
Case B. If the Cauchy data satisfy only $\stackrel{\circ}{G}_{\mu}^{0}=0$, there exists an $H^{s+1}$ coordinate transformation $f$ such that $\frac{\circ}{g}=\left(f^{-1}\right)^{*} \stackrel{\circ}{g}$ has Cauchy data satisfying $\dot{\mathrm{G}}_{\mu}^{0}=0, \stackrel{\circ}{\Gamma}^{\mu}=0$. Hence we get an $H^{s}$ solution $g=f^{*} \bar{g}$ of $R_{\mu \nu}=0$.

Remark. $f$ is not unique, nor is the solution of $R_{\mu \nu}=0$ unique. Case B above constructs a particular one. See the next section for elaboration on this point.

Proof. The system $R_{\mu \nu}^{(h)}=0$ is reduced to a first order system by introducing the new unknowns $g_{\mu \nu}, k_{\mu v}=\partial g_{\mu v} / \partial t, g_{\mu v, i}=\partial g_{\mu v} / \partial x^{i}$, and considering the following first order quasi-linear (symmetric hyperbolic) system of fifty equations:

$$
\left.\begin{array}{rl}
\frac{\partial g_{\mu v}}{\partial t} & =k_{\mu v} \\
g^{i j} \frac{\partial g_{\mu v, i}}{\partial t} & =g^{i j} \frac{\partial k_{\mu v}}{\partial x^{i}} \\
-g^{00} \frac{\partial k_{\mu v}}{\partial t} & =2 g^{0 j} \frac{\partial k_{\mu v}}{\partial x^{j}}+g^{i j} \frac{\partial g_{\mu v, i}}{\partial x^{j}}-2 H_{\mu v}
\end{array}\right\}
$$

where $H_{\mu \nu}\left(g_{\mu \nu}, g_{\mu v, i}, k_{\mu \nu}\right)$, defined earlier, is homogeneous quadratic in $\left(g_{\mu v, i}, k_{\mu \nu}\right)$ and rational in $g_{\mu \nu}$ with non-zero denominator det $g \neq 0$.

Within class $C^{2}$, the system (F) is equivalent to $R_{\mu \nu}^{(h)}=0$. Since $g^{i j}$ is a non-singular matrix (with inverse $g_{k j}-g_{k 0} g_{j 0} / g_{00}$ ) the equations $g^{i j} \frac{\partial g_{\mu v, i}}{\partial t}=g^{i j} \frac{\partial k_{\mu v}}{\partial x^{i}}$ are the same as $\partial g_{\mu v, i} / \partial t=\partial k_{\mu v} / \partial x^{i}$. The first equation than gives $\frac{\partial}{\partial t}\left(g_{\mu v, i}-\partial g_{\mu v} / \partial x^{i}\right)=0$. But equality at $t=0$ gives $g_{\mu v, i}=\partial g_{\mu \nu} / \partial x^{i}$. The last equation is then exactly $R_{\mu \nu}^{(h)}=0$.

We shall now apply Theorem 2.3 to this system $F$ with $s^{\prime}=s-1$ in place of $s$. Let $u=\left(g_{\mu v}, g_{\mu v, i}, k_{\mu v}\right)$ be our fifty component unknown. We specify the closed subspace $F^{s^{\prime}-1}$ and $w$ as follows: $w=\left(\mathbf{1}_{m}, 0,0\right)$ for $\mathbf{1}_{m}$ as defined above and

$$
F^{s^{\prime}-1}=\left\{u \mid \frac{\partial g_{\mu v}}{\partial x^{l}}=g_{\mu v, i}\right\}
$$

which is clearly a closed subspace of $H_{w}^{s^{\prime}-1}\left(\boldsymbol{R}^{3}, \boldsymbol{R}^{50}\right)$. Moreover, the above shows that it is compatible with the equations. Let $U^{s^{\prime}-1}$ be an open set in $H_{w}^{s^{\prime}-1}$ defined such that all denominators are bounded away from zero; for example $U^{s^{\prime}-1}=\left\{u \mid g_{i j}\right.$ is positive definite $g_{00}>\frac{1}{2} \stackrel{\circ}{g}_{00}$, $\left.\operatorname{det} g_{i j}>\frac{1}{2} \operatorname{det} \stackrel{\circ}{g}_{i j}\right\}$. 
The matrices $A^{0}, A^{i}, B$ are given by

$$
\begin{aligned}
& A^{0}\left(g_{\mu v}, g_{\mu v, i}, k_{\mu v}\right)=\left(\begin{array}{ccccc}
I^{10} & 0^{10} & 0^{10} & 0^{10} & 0^{10} \\
0^{10} & g^{11} I^{10} & g^{12} I^{10} & g^{13} I^{10} & 0^{10} \\
0^{10} & g^{12} I^{10} & g^{22} I^{10} & g^{23} I^{10} & 0^{10} \\
0^{10} & g^{13} I^{10} & g^{23} I^{10} & g^{33} I^{10} & 0^{10} \\
0^{10} & 0^{10} & 0^{10} & 0^{10} & -g^{00} I^{10}
\end{array}\right), \\
& A^{j}\left(g_{\mu v}, g_{\mu v, i}, k_{\mu v}\right)=\left(\begin{array}{ccccc}
0^{10} & 0^{10} & 0^{10} & 0^{10} & 0^{10} \\
0^{10} & 0^{10} & 0^{10} & 0^{10} & g^{j 1} I^{10} \\
0^{10} & 0^{10} & 0^{10} & 0^{10} & g^{j 2} I^{10} \\
0^{10} & 0^{10} & 0^{10} & 0^{10} & g^{j 3} I^{10} \\
0^{10} & g^{1 j} I^{10} & g^{2 j} I^{10} & g^{3 j} I^{10} & 2 g^{j 0} I^{10}
\end{array}\right),
\end{aligned}
$$

where $0^{10}$ is the $10 \times 10$ zero matrix, $I^{10}$ is the $10 \times 10$ identity,

$$
B\left(g_{\mu v}, g_{\mu v, i}, k_{\mu v}\right)=\left(\begin{array}{c}
k_{\mu v} \\
0^{30} \\
-2 H_{\mu \nu}\left(g_{\mu v}, g_{\mu v, i}, k_{\mu \nu}\right)
\end{array}\right)=B(u)
$$

and where $0^{30}$ is the thirty component zero vector.

We see that the symmetric hyperbolic system

is just the system $(\mathrm{F})$.

$$
A^{0}(u) \frac{\partial u}{\partial t}=A^{j}(u) \frac{\partial u}{\partial x^{j}}+B(u)
$$

The asymptotic matrices $\tilde{A}_{t}^{0}, \tilde{A}_{t}^{i}, \tilde{B}_{t}$ are just the matrices $A_{t}^{0}, A_{t}^{t}, B_{t}$ with $\mathbf{I}_{m}$ replacing $g_{\mu v}$. It remains to check conditions $\left(\tilde{A}_{2}\right)$. The condition (iii) results from the way we defined $F^{s^{\prime}-1}$, because $u \in F^{s^{\prime}-1}$ implies $g_{\mu \nu} \in H_{w}^{s}$ and $A^{0}, A^{i}, B$ are rational combinations of $g_{\mu \nu}$. From the fact that $H^{s}$ is a ring, we see that condition (iv) is satisfied. Condition (v) is clear. For condition (vi) we can, as in Theorem 1.8, see that the domain of $v \mapsto A^{j}(u) \partial v / \partial x^{j}$ is $H^{s-1} \oplus G^{s^{\prime}} \oplus H^{s^{\prime}}$ and on $F^{s^{\prime}-1}$ the graph norm is $H^{s^{\prime}+1} \oplus H^{s^{\prime}} \oplus H^{s^{\prime}}$ as was shown in Theorem 1.8. Thus this graph norm is independent of $t, u$. Finally, using the $H^{s^{\prime}+1} \oplus H^{s^{\prime}} \oplus H^{s^{\prime}-1}$ norm as $\|\mid \cdot\|_{s}$, we see that (vii) holds as again the coefficients are rational functions of $g_{\mu v}$, and $H^{s^{\prime}}$ is a ring (as long as denominators are bounded $a$ priori away from zero, division by an $H^{s^{\prime}}$ function gives us another $H^{s^{\prime}}$ function).

Thus we have a solution existing in the domain and hence in $H^{s^{\prime}+1}$ $\oplus H^{s^{\prime}} \oplus H^{s^{\prime}}$ and thus we obtain our unique $H^{s}$ spacetime $g_{\mu \nu}$ satisfying $R_{\mu \nu}^{(h)}=0$.

If the Cauchy data satisfies $\stackrel{\circ}{G}_{\mu}^{0}=0, \stackrel{\circ}{\Gamma}^{\mu}=0$, then the conditions of Lemma 3.2 hold and so Case A is established. 
Now we turn to Case B. This is based on the following computation [3]:

3.4. Lemma. Let $s>n / 2+2$ and $g_{\mu v}\left(t, x^{i}\right)$ be an $H^{s}$ spacetime on $V_{4}=I \times \boldsymbol{R}^{3}$ with Cauchy data $\stackrel{\circ}{\mu \nu}_{\mu v}, \partial \stackrel{\circ}{g}_{\mu v} / \partial t$ that satisfies $\dot{G}_{\mu}^{0}=0$. Let $f(x) \equiv \bar{x}^{\mu}\left(x^{\nu}\right)$ be an $H^{s+1}$ coordinate transformation which satisfies:

(i) $\left(\bar{t}\left(0, x^{j}\right), \bar{x}^{i}\left(0, x^{j}\right)\right)=\left(0, x^{j}\right)$,

(ii) $\frac{\partial \bar{x}^{\mu}}{\partial t}\left(0, x^{j}\right)=(1,0)$, and

(iii) $\frac{\partial^{2} \bar{x}^{\mu}}{\partial t^{2}}\left(0, x^{j}\right)=\frac{1}{g^{00}\left(0, x^{j}\right)} \Gamma^{\mu}\left(0, x^{j}\right)$.

Such coordinate transformations exist. Then the Cauchy data of the transformed metric $\bar{g}$ satisfy:

$$
\begin{aligned}
& \stackrel{\circ}{g}_{\alpha \beta}=\stackrel{\circ}{g}_{\alpha \beta} \\
& \frac{\partial \stackrel{\circ}{g}_{i j}}{\partial \bar{t}}=\frac{\partial \stackrel{\circ}{g}_{i j}}{\partial t} \\
& \frac{\partial \stackrel{\circ}{g}_{0 i}}{\partial \bar{t}}=\frac{\partial \stackrel{\circ}{g}_{0 i}}{\partial t}+\stackrel{\circ}{g}_{i \alpha} \frac{\partial^{2} \stackrel{\circ}{x}^{\alpha}}{\partial t^{2}}=\frac{\partial \stackrel{\circ}{g}_{0 i}}{\partial t}+\frac{\stackrel{\circ}{g}_{i \alpha}}{\dot{\circ}^{00}} \stackrel{\circ}{\Gamma}^{\alpha} \\
& \frac{\partial \stackrel{\circ}{g}_{00}}{\partial \bar{t}}=\frac{\partial \stackrel{\circ}{g}_{00}}{\partial t}+\stackrel{\circ}{g}_{0 \alpha} \frac{\partial^{2} \bar{x}^{\alpha}}{\partial t^{2}}=\frac{\partial \stackrel{\circ}{g} 00}{\partial t}+\frac{\stackrel{\circ}{0 \alpha}_{0 \alpha}}{\dot{o}^{00}} \stackrel{\circ}{\Gamma}^{\alpha}
\end{aligned}
$$

and

$$
\stackrel{\circ}{\Gamma}^{\mu}=0, \quad \stackrel{\circ}{G}_{\mu}=0 .
$$

Existence of $f$ is established in $\S 4$. The existence of $\bar{x}^{\mu}$ is slightly delicate because the right hand side of (iii) is just $H^{s-1}$. We shall obtain the existence of such transformations in the next section by solving a suitable wave equation. The rest of the assertions can be checked by a straightforward computation using the transformation rule for $\Gamma^{\mu}$ :

$$
\bar{\Gamma}^{\mu}=\frac{\partial \bar{x}^{\mu}}{\partial x^{\alpha}} \Gamma^{\alpha}-g^{\alpha \beta} \frac{\partial^{2} \bar{x}^{\mu}}{\partial x^{\alpha} \partial x^{\beta}}
$$

and the fact that $G_{\mu}^{0}$ does not depend on $\partial g_{0 \mu} / \partial t$.

To complete Case B we consider the solution $\bar{g}_{\mu \nu}$ of $\bar{R}_{\mu \nu}^{(h)}=0$ with Cauchy data given by the lemma in the $\bar{x}^{\alpha}$ system. By case $A, \bar{g}_{\mu \nu}$ satisfies $\bar{R}_{\mu \nu}=0$. Hence $g=f^{*} \bar{g}$ will also satisfy $R_{\mu \nu}=0$ (since it is a tensorial equation) and will have the originally given Cauchy data. By Lemma 3.1, $g$ satisfies the asymptotic and smoothness properties of a spacetime. This completes the proof. 


\section{§ 4. Uniqueness for the Einstein System}

In this section we shall show that any two $H^{s}$ asymptotically flat spacetimes which are Ricci flat and have the same Cauchy data are related by an $H^{s+1}$ coordinate transformation asymptotic to the identity. A local result like this with weaker differentiability of the coordinate transformation was obtained by Choquet-Bruhat [6]. See also ChoquetBruhat and Geroch [11] where the problem of maximal extendability in spacetimes is discussed.

Our proof is based on Theorem 1.8 which tells us that for the wave equation with $\left(H^{s}, H^{s-1}\right)$ coefficients, solutions exist in the class $\left(H^{s+1}, H^{s}\right)$.

As is well known, solutions to the system $R_{\mu \nu}$ are not functionally unique. Thus if $g_{\mu \nu}$ is an $H^{s}$ asymptotically flat solution to $R_{\mu \nu}=0$ and $\bar{x}^{\mu}=\bar{x}^{\mu}\left(x^{\nu}\right)$ is an $H^{s+1}$ coordinate transformation asymptotic to the identity that is the identity on the surface $t=0, \frac{\partial \bar{x}^{\mu}}{\partial t}\left(0, x^{\nu}\right)=\delta_{0}^{\mu}$ and $\frac{\partial^{2} \bar{x}^{\mu}}{\partial t^{2}}\left(0, x^{i}\right)=0$, then by covariance of $R_{\mu \nu}=0$ and by Lemma 3.1 , $\bar{g}_{\mu \nu}\left(\bar{x}^{\lambda}\right)=g_{\alpha \beta}\left(x^{\varrho}\left(\bar{x}^{\lambda}\right)\right) \frac{\partial x^{\alpha}}{\partial \bar{x}^{\mu}}\left(\bar{x}^{\lambda}\right) \frac{\partial x^{\beta}}{\partial \bar{x}^{\nu}}\left(\bar{x}^{\lambda}\right)$ is also an $H^{s}$ asymptotically flat solution to $R_{\mu \nu}=0$ and which has the same Cauchy data as $g_{\mu \nu}\left(x^{\varrho}\right)$. Of course, a similar situation holds for any tensor system of partial differential equations on a spacetime.

Thus we want to prove the converse of the above remark. That is, two $H^{s}$ solutions $g, \bar{g}$ with the same Cauchy data are $H^{s+1}$ isometric. As a first step, we shall now prove that when we bring an $H^{s}$ spacetime to harmonic coordinates, it remains $H^{s}$. To do this requires an $H^{s+1}$ coordinate transformation. This will also complete the existence part of Theorem 3.3, Case B.

4.1. Theorem. Let $g_{\mu \nu}$ be an $H^{s}$ spacetime, $s \geqq 4$, on $I \times \boldsymbol{R}^{3}, I=(-\varepsilon, \varepsilon)$. Then there is an $\varepsilon^{\prime}, 0<\varepsilon^{\prime} \leqq \varepsilon$ and a unique $H^{s+1}$ coordinate transformation $\phi: I^{\prime} \times \boldsymbol{R}^{3} \rightarrow I \times \boldsymbol{R}^{3} \quad x^{\underline{Q}} \mapsto \bar{x}^{\mu}=\phi\left(x^{o}\right)$ asymptotic to the identity such that the transformed metric $\bar{g}_{\mu v}$ is an $H^{s}$ spacetime with $\bar{\Gamma}^{\mu}\left(\bar{x}^{\lambda}\right)=0$.

Proof. Consider the wave equation for the scalar $\psi$,

$$
\square \psi=-g^{\alpha \beta}-\frac{\partial^{2} \psi}{\partial x^{\alpha} \partial x^{\beta}}+g^{\alpha \beta} \Gamma_{\alpha \beta}^{\mu} \frac{\partial \psi}{\partial x^{\mu}}=0 .
$$

The coefficients are of class $H^{s}, H^{s-1}$. Let $\bar{t}(t, x)$ be the unique solution with Cauchy data

$$
\bar{t}\left(0, x^{j}\right)=0, \quad \frac{\partial \bar{t}}{\partial t}\left(0, x^{j}\right)=1 .
$$


To fit this into the framework of Theorem 1.8, consider instead the equation for $\xi(t, x)=\bar{t}(t, x)-t$ which is

$$
\left\{\begin{array}{l}
-g^{\alpha \beta} \frac{\partial^{2} \xi}{\partial x^{\alpha} \partial x^{\beta}}+g^{\alpha \beta} \Gamma_{\alpha \beta}^{\mu} \frac{\partial \xi}{\partial x^{\mu}}+g^{\alpha \beta} \Gamma_{\alpha \beta}^{0}=0 \\
\xi(0, x)=0, \quad \frac{\partial \xi}{\partial t}(0, x)=0 .
\end{array}\right.
$$

Note that the coefficients are $H^{s}, H^{s-1}$ and the inhomogeneous term is $H^{s-1}$. Thus by 1.8 we get a solution $\xi$ in $H^{s+1}$, and hence have defined a function $\bar{t}(t, x) H^{s+1}$ asymptotic to $t$.

In a similar way we may use theorem 1.8 to solve $\square \psi=0$ for $\bar{x}^{i}$ with Cauchy data

$$
\bar{x}^{i}\left(0, x^{j}\right)=x^{j}, \quad \frac{\partial \bar{x}^{i}}{\partial t}\left(0, x^{j}\right)=0 .
$$

Now $t \mapsto \bar{x}^{i}(t, \cdot)$ is continuous in $H^{s+1}$ (by Theorem 1.8) and $\mathscr{D}_{I}^{s+1}$ is open in $H^{s+1}$ (Cantor [5]) so $\bar{x}^{i}$ is an $H^{s+1}$ diffeomorphism of $\boldsymbol{R}^{3}$ to $\boldsymbol{R}^{3}$ for some $t$-interval. Also, $\bar{t}$ is for fixed $x$ a diffeomorphism in $t$ (on a uniform $t$-interval for all $x$ since $\partial \bar{t} / \partial t=1$ ) by the implicit function theorem. Thus $f(t, x)=(\bar{t}(t, x), \bar{x}(t, x))$ on $I^{\prime} \times \boldsymbol{R}^{3}$ is an $H^{s+1}$ coordinate transformation asymptotic to the identity, so that by Lemma $3.1 \bar{g}=\left(f^{-1}\right)^{*} g$ is an $H^{s}$ spacetime.

Now the equation $\square \bar{x}^{\mu}=0$ is a tensorial (scalar) equation. In the barred coordinates, it becomes

$$
\begin{aligned}
0 & =\bar{\square} \bar{x}^{\mu} \\
& =-\bar{g}^{\alpha \beta} \frac{\partial^{2} \bar{x}^{\mu}}{\partial \bar{x}^{\alpha} \partial \bar{x}^{\beta}}+\bar{\Gamma}^{\alpha} \frac{\partial \bar{x}^{\mu}}{\partial \bar{x}^{\alpha}} \\
& =\bar{\Gamma}^{\mu}
\end{aligned}
$$

so that $\bar{g}_{\mu v}$ satisfies the harmonic condition. Thus $f$ transforms $g_{\mu v}$ to harmonic coordinates. Uniqueness of $f$ follows from uniqueness of solutions to the wave equation with given Cauchy data.

Note that since $g_{\mu \nu}$ is of class $H^{s}$, the sharpness of Theorem 1.8 is actually needed to show that the transformation $f$ is $H^{s+1}$ and thus that $\bar{g}_{\mu \nu}$ in harmonic coordinates is also $H^{s}$. Note also that we have uniqueness of $f$ because the Cauchy data used to solve the wave equation for harmonic coordinates is contained in our definition of coordinate transformation. Moreover, since $f$ leaves the hyperplane $t=0$ fixed and $D f$ is the identity on $t=0, f$ cannot even be composed with an isometry, as an isometry which fixes a point and a frame at that point is the identity. 
4.2. Theorem. Let $g_{\mu \nu}^{(1)}$ and $g_{\mu \nu}^{(2)}$ be two $H^{s}$ spacetimes on $(-\varepsilon, \varepsilon) \times \boldsymbol{R}^{3}$ with the same Cauchy data and which satisfy $R_{\mu \nu}=0$.

Then there is an $\varepsilon^{\prime}, 0<\varepsilon^{\prime} \leqq \varepsilon$ and a unique $H^{\text {s+1 }}$ coordinate transformation $\phi: I^{\prime}=\left(-\varepsilon^{\prime}, \varepsilon^{\prime}\right) \times \boldsymbol{R}^{3} \rightarrow I \times \boldsymbol{R}^{3}$ asymptotic to the identity such that $g^{(2)}=\left(\phi^{-1}\right)^{*} g^{(1)}$ on $\left(-\varepsilon^{\prime}, \varepsilon^{\prime}\right) \times \boldsymbol{R}^{3}$.

Proof. From Theorem 4.1, we can find the unique $H^{s+1}$ coordinate transformations $\phi_{1}$ and $\phi_{2}$ such that $\bar{g}^{(1)}=\left(\phi_{1}^{-1}\right)^{*} g^{(1)}$ and $\bar{g}^{(2)}=\left(\phi_{2}^{-1}\right)^{*} g^{(2)}$ satisfy the harmonic condition. Moreover, from the expression for the transformed Cauchy data and from the equality of the Cauchy data of $g^{(1)}$ and $g^{(2)}$, we see that the Cauchy data of $\bar{g}^{(1)}$ and $\bar{g}^{(2)}$ are also equal. But both $\bar{g}^{(1)}$ and $\bar{g}^{(2)}$ then satisfy $R_{\mu \nu}^{(h)}=0$ with the same Cauchy data, so from uniqueness for that system, $\bar{g}^{(1)}=\bar{g}^{(2)}$ (on their common $t$-interval). Thus $\left(\phi_{1}^{-1}\right)^{*} g^{(1)}=\left(\phi_{2}^{-1}\right)^{*} g^{(2)}$, or

$$
g^{(2)}=\left(\phi^{-1}\right)^{*} g^{(1)}
$$

where $\phi=\phi_{2} \circ \phi_{1}^{-1}$. It follows from the definitions and the fact that $\mathscr{D}_{I}^{s+1}$ is a topological group that $\phi$ satisfies the conditions for an $H^{s+1}$ coordinate transformation asymptotic to the identity. That $\phi$ is unique follows from uniqueness of $\phi_{1}$ and $\phi_{2}$.

We showed above that given $H^{s}, H^{s-1}$ Cauchy data, the system $R_{\mu v}=0$ always has some $H^{s}$ solution. However, one may desire that the solution have $g_{00}, g_{0 i}$ specified; for example, in Gaussian coordinates with $g_{00}=-1, g_{0 i}=0$. In this case, it seems that the best one can do is to obtain an $H^{s-2}$ spacetime from $H^{s}, H^{s-1}$ initial data. The case of general $g_{00}$ and $g_{0 i}$ is similar; see [20].

4.3. Theorem. Let $s>n / 2+3(n=3)$. Then for $\left(\stackrel{\circ}{g}, \frac{\partial \dot{g}}{\partial t}\right) \in \mathscr{L}^{s} \times \mathscr{K}^{s-1}$ which satisfies $\stackrel{\circ}{g}_{00}=-1, \stackrel{\circ}{g}_{0 i}=0, \partial \stackrel{\circ}{g}_{0 u} / \partial t=0$, there exists a unique $H^{s-2}$ spacetime $g$ with this Cauchy data, satisfying $g_{00}=-1, g_{0 i}=0$ and $R_{\mu \nu}=0$, for $-\varepsilon<t<\varepsilon, \varepsilon>0$.

Proof. Consider some $H^{s}$ spacetime $\tilde{g}_{\mu v}$ with the given Cauchy data and satisfying $R_{\mu v}=0$, whose existence was proved in 3.3. Consider now the geodesic ordinary differential equations defined by $\tilde{g}$; that is, consider the spray of $\tilde{g}([30,34])$. This is a system of ordinary differential equations with $H^{s-1}$ coefficients:

$$
\left\{\begin{array}{l}
\frac{d x^{\alpha}}{d t}=v^{\alpha} \\
\frac{d v^{\alpha}}{d t}=-\Gamma_{\beta \gamma}^{\alpha} v^{\beta} v^{\gamma} .
\end{array}\right.
$$


Since we have conservation of energy we can multiply $v$ by a $C^{\infty}$ function of $\|v\|$ with compact support $\|v\| \leqq M$ without affecting solutions with $\|v\| \leqq M$. Then we get a system $\frac{d x}{d t}=X_{1}(x, v), \frac{d v}{d t}=X_{2}(x, v)$ where $X_{1}, X_{2}$ are $H^{s-1}$ functions. By Appendix B (sharpening a result of EbinMarsden [19]), this has an $H^{s-1}$ flow asymptotic to the identity. Thus, the associated exponential map will be an $H^{s-1}$ diffeomorphism $\phi(t, x)$ asymptotic to the identity. This $\phi$ satisfies the requirements of an $H^{s-1}$ coordinate transformation. Thus $\phi^{-1 *} \tilde{g}=g$ will be an $H^{s-2}$ spacetime which, as is well known (cf. Synge [41]), satisfies $g_{00}=-1, g_{0 i}=0$. This proves the existence part of 4.3. Note: $g$ satisfies $R_{\mu \nu}=0$ in a distributional sense; cf. 3.2. For $g$ to be defined we only need $s>n / 2+2$; for $R_{\mu \nu}=0$ to make sense, $g$ should be at least $C^{1}$ and $H^{2}$ and so $s>n / 2+3$.

For uniqueness, we may prove, as in Theorem 4.3, that if $g^{1}$ and $g^{2}$ are two $\mathrm{H}^{s-2}$ solutions, then there exists an $\mathrm{H}^{s-1}$ coordinate transformation $\phi$ (asymptotic to the identity) such that $g^{(2)}=\left(\phi^{-1}\right)^{*} g^{(1)}$. Thus $g^{(1)}$ is obtained from $g^{(2)}$ by a coordinate transformation. But from the theory developed in [20], it follows that such a coordinate transformation mapping one solution of the Einstein system to another with the same Cauchy data is completely determined by the lapse and shift function. Since they are prescribed here, $g^{(1)}=g^{(2)}$. One can also see this directly by writing out $\left(\phi^{-1}\right)^{*} g^{(1)}=g^{(2)}$ and using the conditions $g_{00}^{(A)}=-1$, $g_{0 i}^{(A)}=0, A=1,2$.

The same sort of argument works to show existence of unique solutions for a general lapse and shift. Finally we remark that it seems quite difficult to prove such results directly from the equations in the dynamical $3+1$ formulation (cf. [20]).

Remark. In future papers we plan to discuss the question of global existence of non-singular spacetimes. Besides the above existence theory, the existence of such spacetimes depends on (a) finding non-trivial solutions to the constraint equations near the flat solution which depend on only one variable (we believe that toroidal gravitational waves are examples), (b) the use of the mass function introduced by Brill and Deser (Ann. Phys. 7, 548 (1968)) and positive definiteness of its second variation. See J. Marsden and A. Fischer, Publications du départément de mathématiques, Université de Lyon; Lecture, March 3, 1972.

\section{Appendix A. Smoothness of Non-Linear Semi-Groups}

Here we prove a general result on the smoothness of a non-linear semigroup $F_{t}$ for fixed $t$ and apply it to the case of symmetric hyperbolic systems (Theorem 2.6). The smoothness for fixed $t$ is important in many applications; cf. [13]. 
Let $D \subseteq \boldsymbol{E}$ be Banach spaces and $X: D \rightarrow \boldsymbol{E}$ a $C^{1}$ map. By a flow for $X$ we mean continuous maps $F_{t}: D \rightarrow D$ such that $F_{0}(x)=x, F_{t+s}=F_{t} \circ F_{s}$ and $\frac{d}{d t} F_{t}(x)=X\left(F_{t}(x)\right)$ with the derivative as a curve in $\boldsymbol{E}$. The flow $F_{t}$ need in general only be locally defined and for $t \geqq 0$.

Recall that a linear semi-group $U_{t}$ is called quasi-contractive iff $\left\|U_{t}\right\| \leqq e^{\beta t}$ for a constant $\beta \geqq 0$.

Theorem. Let $X: D \rightarrow \boldsymbol{E}$ be of class $C^{1}$ with a flow $F_{t}: D \rightarrow D$. For $x \in D$ let $Y_{x}$ be the part of the linear operator $D X(x): D \rightarrow \boldsymbol{E}$ in $D\left(Y_{x}=D X(x)\right.$ on the domain $=\{y \mid D X(x) \cdot y \in D\})$. Suppose

(a) $Y_{x}$ generates a quasi-contractive semi-group on $D$ with constant $\beta$ locally bounded in $x \in D$

(b) the domain of $Y_{x}$ is independent of $x \in D$.

For each $u, v \in D$ write

$$
X(u)-X(v)=Z(u, v) \cdot(u-v)
$$

$\left(\right.$ so $\left.Z(u, v)=\int_{0}^{1} D X(s u+(1-s) v) d s\right)$ and also let $Z$ stand for its part in $D$. Assume, more generally than (a), (b) that:

(c) The domain of $Z$ equals that of $Y_{x}$,

(d) $Z$ is strongly continuous as an operator in $D$ as u varies in $D$, locally uniformly in $u \in D$ (note that $Z(u, u)=Y_{u}$ ), and

(e) $Z(u, v)$ generates a quasi-contractive semi-group with $\beta$ locally bounded in $u, v$.

Then for each fixed $t, F_{t}: D \rightarrow D$ is differentiable with strongly continuous derivative, locally uniformly bounded.

Note. If one is willing to add more differentiability in $u, v$, condition (d) can be relaxed to resolvent continuity.

Of course one can iterate the process. Sometimes one says $F_{t}$ is of class $B T^{1}$ for the conclusions here. If $D F_{t}$ is again of this type, $F_{t}$ is $C^{1}$ as is easy to see (see Abraham [1]).

The proof of the theorem is based on Kato's theory of time-dependent evolution equations; Kato [26-28].

Namely, we can find an evolution operator for the family of timedependent operators $A(t)=Y_{F_{t}(x)}$ for $x \in D$ fixed. This is possible because $A(t)$ are all quasi-contractive (hence "stable") and have a fixed domain and vary continuously in $t$. Call this evolution operator $H_{t}: D \rightarrow D$ (starting at $s=0$ ). What we claim is that $D F_{t}(x)=H_{t}$. To see this, we note 
that $\theta_{t}(h)=\frac{1}{\|h\|}\left(F_{t}(x+h)-F_{t}(x)\right)$ solves the equation

$$
\begin{aligned}
\frac{d}{d t} \theta_{t}(h) & =\left[X\left(F_{t}(x+h)\right)-X\left(F_{t}(x)\right)\right] /\|h\| \\
& =Z\left(F_{t}(x+h), F_{t}(x)\right) \cdot \theta_{t}(h)
\end{aligned}
$$

and $\theta_{0}(h)=h /\|h\|=e$, say. Now the operators $A_{h}(t)=Z\left(F_{t}(x+h), F_{t}(x)\right)$ also generate evolution operators and $\theta_{t}(h)$ is an integral curve. As $h \rightarrow 0$, $A_{h}(t) \rightarrow A(t)$ strongly for each $t$. Thus by Kato [28], the solutions with fixed initial data $e$ also converge, as $h \rightarrow 0$. Thus as $h \rightarrow 0$ we get

$$
\frac{F_{t}(x+h)-F_{t}(x)}{\|h\|} \rightarrow H_{t} \cdot h /\|h\| \text {. }
$$

The convergence is uniform over the directions of $h$ because of (d). Thus $D F_{t}(x)$ exists and equals $h \mapsto H_{t} \cdot h$. This derivative is strongly continuous and locally bounded in $x$ because $Y_{F_{t}(x)}$ is. This proves the theorem.

To apply the theorem to the symmetric hyperbolic case, we consider the situation of, say 2.1, with $D=D^{s}$ and $\boldsymbol{E}=H^{s}$. The non-trivial part of the hypotheses is to verify (d) with $u, v$ varying in the $D^{s}$ topology. To see this, we may take $A^{0}=I$ and confine ourselves to the $A$ term, so $X(u)=A^{i}(u) \frac{\partial u}{\partial x^{i}}$. Now write

$$
X(u)-X(v)=A^{i}(u) \frac{\partial(u-v)}{\partial x^{i}}+\left[A^{i}(u)-A^{i}(v)\right] \frac{\partial v}{\partial x^{i}} .
$$

We may write $A^{i}(u)-A^{i}(v)=R^{i}(u, v) \cdot(u-v)$ where $R^{i}$ is, say, some algebraic combination of $u, v$. Thus we take

$$
Z(u, v)=A^{i}(u) \frac{\partial}{\partial x^{i}}+\frac{\partial v}{\partial x^{l}} R^{i}(u, v) .
$$

The last term is like a $B$ term in our symmetric hyperbolic system and the first term has coefficients depending continuously on $u \in D^{s}$. Thus $Z(u, v)$ depends continuously on $u \in D^{s}$. This shows that 2.6 is a special case of the above theorem.

\section{Appendix B. Flows of $H^{s}$ Vector Fields}

Theorem. Let $X: \boldsymbol{R}^{n} \rightarrow \boldsymbol{R}^{n}$ be an $H^{s}$ vector field (asymptotic to zero) where $s>n / 2+1$. Then $X$ has a flow $F_{t} \in \mathscr{D}_{I}^{s}\left(F_{t}\right.$ is defined by $\frac{d}{d t} F_{t}(x)$ $=X\left(F_{t}(x)\right), x \in \boldsymbol{R}^{n}$ and $\mathscr{D}_{I}^{s}$ are the $H^{s}$ diffeomorphisms asymptotic to the identity). 
This improves a result of Ebin-Marsden [19] in two ways. First, we have lowered the permissible value of $s$ by one, and second we are working in a non-compact setting.

A careful examination of the proof in [19] together with the results of Cantor [5] can, in fact, be used to prove the theorem. The result has been noted independently by $\mathrm{H}$. Brezis who also remarks that the result is true generally for $X$ of class $W^{s, p}, s>\frac{n}{p}+1$. However, we can give an entirely different argument using the theory of symmetric hyperbolic systems.

Proof of Theorem. Since $s>n / 2+1$, it follows that the $C^{1}$ norm of $X$ is uniformly bounded. Hence $X$ has, by classical theory [30], a globally defined $C^{1}$ flow $F_{t}$. What we shall show is that $F_{t}-$ Id lies in $H^{s}$. Since $F_{t}$ is invertible, this will prove the assertion. Consider the scalar equation

$$
\frac{\partial \phi}{\partial t}=\Sigma X^{i}(x) \frac{\partial \phi}{\partial x^{i}}
$$

with $\phi(0, x)=\phi_{0}(x)$ given. As is well known, this has solution

$$
\phi(t, x)=\phi_{0} \circ F_{t}(x)
$$

(this is just the method of characteristics for first order partial differential scalar equations).

Now (1) is also a symmetric hyperbolic system with $H^{s}$ coefficients, and so we may apply the theory in $\S 1$. Thus $\phi_{0}$ of class $H^{s}$ implies $\phi_{t}$ is $H^{s}$.

In particular let $\phi_{0}(x)=x^{i}$, the projection on the $i^{\text {th }}$ coordinate. The equation for is

$$
\begin{gathered}
\xi=\phi-\phi_{0} \\
\frac{\partial \xi}{\partial t}=\Sigma X^{j}(x) \frac{\partial \xi}{\partial x^{j}}+X^{i}(x) \\
\xi(0)=0 .
\end{gathered}
$$

By (the inhomogeneous) Theorem 1.1, the solution $\xi(t, x)$ is in $H^{s}$. Hence we have

$$
F_{t}^{i}(x)-x^{i} \text { is } H^{s}
$$

(asymptotic to zero). This means $F_{t}$ - Id is $H^{s}$ and completes the proof.

To extend this to non-flat manifolds requires the same sort of completeness and curvature assumptions which is needed for the noncompact $H^{s}$ theory. These details will appear in Part II.

Acknowledgements. We thank H. Brezis, Y. Choquet-Bruhat, M. Cantor, D. Ebin, K. O. Friedrichs, T. Kato, H. Künzle, P. Lax, H. Levy, A. Lichnerowicz, M. Protter, R. Sachs, and A. Taub for several helpful discussions. 
Note added in proof. (a) Refinements of some of the results in $\S \S 1,2$ have been obtained by $\mathrm{T}$. Kato, using the methods of [29].

(b) Strictly speaking, in 1.8 we should work in the closed subspace $F^{s}$ of those $u$ in $H^{\mathrm{s}}$ such that $\psi_{i}=\partial \psi / \partial x^{i}$. This makes the domains independent of $t$ and is why we use $F^{s}$ in $\left(\tilde{A}_{2}\right)$ and $\left(\tilde{A}_{3}\right)$ on pp. 13 and 18 .

(c) Some existence theorems for the nonlinear constraint equations will appear in a forthcoming paper.

\section{References}

1. Abraham, R.: Lectures on global analysis. Mimeographed, Princeton University.

2. Arnowitt, R., Deser, S., Misner, C.W.: The dynamics of general relativity. In: Witten, L. (Ed.): Gravitation; an introduction to current research. New York: John Wiley 1962.

3. Fourès-Bruhat, Y.: Théorèm d'existence pour certain systèmes d'équations aux dérivées partielles non linéaires. Acta Math. 88, 141-225 (1952).

4. - Cauchy problem. In: Witten, L. (Ed.): Gravitation; an introduction to current research. New York: John Wiley 1962.

5. Cantor, M.: Diffeomorphism groups over manifolds with non-compact base (preprint).

6. Choquet-Bruhat, Y.: Espaces-temps einsteiniens généraux, chocs gravitationels. Ann. Inst. Henri Poincaré 8, 327-338 (1968).

7. - Solutions $C^{\infty}$ d'équations hyperboliques non linéares. C. R. Acad. Sci. Paris 272, $386-388$ (1971).

8. - Problèmes mathématiques en relativité. Actes Congres intern. Math. Tome 3, 27-32 (1970).

9. - $C^{\infty}$ solutions of hyperbolic non-linear equations; applications in general relativity and gravitation. Gen. Rel. Grav. 1 (1971).

10. - Stabilité de solutions d'équations hyperboliques non linéares. Application à l'espace-temps de Minkowski en relativité générale. C. R. Acad. Sci. Paris 274, Ser. A. (843) (1972).

11. - Geroch, R.: Global aspects of the Cauchy problem in general relativity. Commun. math. Phys. 14, 329-335 (1969).

12. Chernoff, P.: Note on product formulas for operator semigroups. J. Funct. An. 2, $238-242(1968)$.

13. Chernoff, P., Marsden, J.: On continuity and smoothness of group actions. Bull. Am. Math. Soc. 76, 1044-1049 (1970).

14. - - Hamiltonian systems and quantum mechanics (in preparation).

15. Courant, R., Hilbert, D.: Methods of mathematical physics, Vol. II. New York: Interscience 1962.

16. Dionne, P.: Sur les problèmes de Cauchy bien posés. J. Anal. Math. Jerusalem 10, $1-90(1962 / 63)$.

17. Dunford, N., Schwartz, J.: Linear operators (I). New York: Interscience 1962.

18. Ebin, D.: On the space of Riemannian metrics. In: Proc. Symp. Pure Math., vol. 15. Providence, R. I.: Math. Soc. 1970.

19. - Marsden, J.: Groups of diffeomorphisms and the motion of an incompressible fluid. Ann. Math. 92, 102-163 (1970).

20. Fischer, A., Marsden, J.: The Einstein equations of evolution - A geometric approach. J. Math. Phys. 13, 546-568 (1972).

21. - - General relativity, partial differential equations and dynamical systems. In: Proc. Symp. Pure Math., vol. 23. Providence, Rhode Island: Am. Math. Soc. 1972 (to appear).

22. Fock, V.: The theory of space, time and gravitation. New York: MacMillan 1964. 
23. Friedrichs, K.O.: Symmetric hyperbolic linear differential equations. Commun. Pure Appl. Math. 7, 345-392 (1954).

24. - A limiting process leading to the equations of relativistic and nonrelativistic fluid dynamics. In: La Magnétohydrodynamique Classique et Relativiste, Lille 1969. Colloques Internat. CNRS, No. 184, Paris, 1970.

25. Hawking, S.: Stable and generic properties in general relativity. Gen. Rel. Grav. 1, 393-400 (1971).

26. Kato, T.: Perturbation theory for linear operators. Berlin-Heidelberg-New York: Springer 1966.

27. - Integration of the equation of evolution in a Banach space. J. Math. Soc. Japan 5, $208-234$ (1953).

28. - Approximation theorems for evolution equations. In: Aziz, A. (Ed.): Lectures in Differential Equations. Mathematical Studies, Vol. 19. New York: Van Nostrand 1969.

29. - Linear evolution equations of "hyperbolic type". J. Fac. Sci. Univ. Tokyo 17, $241-258(1970)$.

30. Lang, S.: Introduction to differentiable manifolds. New York: Interscience 1962.

31. Lax, P.: Cauchy's problem for hyperbolic equations and the differentiability of solutions of elliptic equations. Commun. Pure Appl. Math. 8, 615-633 (1955).

32. Leray, J.: Lectures on hyperbolic equations with variable coefficients. Princeton, Inst. for Adv. Stud. 1952.

33. Lichnerowicz, A.: Relativistic Hydrodynamics and Magnetohydrodynamics. New York: Benjamin 1967.

34. Marsden,J., Ebin,D., Fischer, A.: Diffeomorphism groups, hydrodynamics and relativity. In: Proc. Canadian Math. Congress XIII, Halifax (1971) (to appear).

35. Nirenberg, L. : On elliptic differential equations. Scuola Norm. Super. Pisa 13, 115-162 (1959).

36. Palais, R.: Seminar on the Atiyah Singer index theorem. Ann. of Math. Studies, no. 57. Princeton: Princeton University Press 1965.

37. Phillips, R.: Dissipative operators and hyperbolic systems of partial differential equations. Trans. Am. Math. Soc. 90, 193-254 (1959).

38. Schulenberger, J., Wilcox, C.: Completeness of the wave operators for perturbations of uniformly propagative systems. J. Funct. An. 7, 447-474 (1971).

39. Segal, I.: Non-linear semi-groups. Ann. Math. 78, 339-364 (1963).

40. Sobolev, S. L.: Applications of functional analysis in mathematical physics. Providence, R. I.: Am. Math. Soc. 1963.

41. Synge,J.L.: Relativity: The general theory. Amsterdam: North-Holland Publ. Co. 1960.

42. Wilcox, C.: The domain of dependence inequality for symmetric hyperbolic systems. Bull. Am. Math. Soc. 70, 149-154 (1964).

43. Yosida, K.: Functional Analysis. Berlin-Heidelberg-New York: Springer 1965.

Arthur E. Fischer

Jerrold E. Marsden

Department of Mathematics

University of California

Berkeley, California 94720, USA 